MATHEMATICS OF COMPUTATION

Volume 70, Number 235, Pages 951-976

S $0025-5718(00) 01287-4$

Article electronically published on October 18, 2000

\title{
A NEW STABILIZING TECHNIQUE FOR BOUNDARY INTEGRAL METHODS FOR WATER WAVES
}

\author{
THOMAS Y. HOU AND PINGWEN ZHANG
}

\begin{abstract}
Boundary integral methods to compute interfacial flows are very sensitive to numerical instabilities. A previous stability analysis by Beale, Hou and Lowengrub reveals that a very delicate balance among terms with singular integrals and derivatives must be preserved at the discrete level in order to maintain numerical stability. Such balance can be preserved by applying suitable numerical filtering at certain places of the discretization. While this filtering technique is effective for two-dimensional (2-D) periodic fluid interfaces, it does not apply to nonperiodic fluid interfaces. Moreover, using the filtering technique alone does not seem to be sufficient to stabilize 3-D fluid interfaces.

Here we introduce a new stabilizing technique for boundary integral methods for water waves which applies to nonperiodic and 3-D interfaces. A stabilizing term is added to the boundary integral method which exactly cancels the destabilizing term produced by the point vortex method approximation to the leading order. This modified boundary integral method still has the same order of accuracy as the point vortex method. A detailed stability analysis is presented for the point vortex method for 2-D water waves. The effect of various stabilizing terms is illustrated through careful numerical experiments.
\end{abstract}

\section{INTRODUCTION}

Boundary integral methods have been one of the commonly used numerical methods in studying the motion of free surfaces. Examples of applications include water waves, vortex sheets, multifluid interfaces, Hele-Shaw cells, and crystal growth and solidification. The advantage of boundary integral methods is that they reduce the dimension of the problem by using quantities along the interface only. As a consequence, this avoids the difficulty of differentiating discontinuous fluid quantities across the fluid interface, and makes it possible to design high-order discretizations for the governing equations. On the other hand, it is also well known that boundary integral methods are very sensitive to numerical instabilities [10, 5, 2, 3, 7.

Received by the editor August 14, 1998 and, in revised form, August 5, 1999.

2000 Mathematics Subject Classification. Primary 65M12, 76B15.

Key words and phrases. Boundary integral method, stability, water waves.

The first author was partially supported by the National Science Foundation under grant DMS-9704976, by the Office of Naval Research under grant N00014-96-1-0438, and by the Army Research Office under grant DAAD19-99-1-0141.

The second author was partially supported by the National Science Foundation under grant DMS-9704976 and by the Office of Naval Research under grant N00014-96-1-0438. He was also supported by the National Natural Science Foundation of China and the China State Major Key Project for Basic Research. 
Numerical errors even at the round-off level may grow rapidly in time. This has been one of the main difficulties of using boundary integral methods in practice.

Beale, Hou, and Lowengrub [3] presented a convergence proof of a spectrally accurate boundary integral method for 2-D water waves with or without surface tension. They found that a certain compatibility is required between the choice of quadrature rules for the singular integrals and the choice for the approximation of the spatial derivative in the evolution equations of the water interface. This compatibility ensures that a delicate balance of terms on the continuous level is preserved on the discrete level. This balance is crucial for maintaining numerical stability. When the water interface is a periodic perturbation of the flat interface, a suitable filtering on the interface position variable, $z(\alpha)$, is introduced by Beale et $a l$. 3 to enforce the discrete compatibility. Here $\alpha$ is a Lagrangian parameter. The filtering in $z(\alpha)$ is done by multiplying by a nonnegative cut-off function, $\rho(k h)$ in the Fourier transform. When $z_{j}$ is an approximation to $z\left(\alpha_{j}\right), s_{j}=z_{j}-\alpha_{j}$ will be periodic. The filtering of $z_{j}$, denoted as $z_{j}^{p}$, is defined as $\alpha_{j}+s_{j}^{p}$, where $\hat{s}_{k}^{p}=\rho(k h) \hat{s}_{k}$, and $\hat{s}_{k}$ is the discrete Fourier transform of $s$ corresponding to wavenumber $k$. The amount of filtering, $\rho$, is determined by the quadrature rule in approximating the integral that gives the velocity and the derivative rule being used (see [3] and the filtering scheme described by equations (46)-(48) in Section 4).

However, the Fourier filtering also has some limitations. It does not apply directly to nonperiodic interfaces. Moreover, for problems without spectrally accurate discretizations for the singular boundary integral, there are insufficient degrees of freedom to enforce all the compatibility conditions by filtering alone. For example, for 3-D free surfaces, the singular kernel has an unremovable branch point singularity. It seems extremely difficult to obtain a spectrally accurate discretization for 3 -D water waves. Furthermore, the singular 3 -D kernel introduces a number of additional compatibility conditions between different singular integral operators and the Lagrangian derivative operator [8]. This makes it almost impossible to enforce the corresponding discrete compatibility conditions by using Fourier filtering alone.

In this paper, we introduce a new stabilizing technique which does not rely on Fourier filtering. A stabilizing term is added to the boundary integral method which exactly cancels the destabilizing term produced by the point vortex method approximation. The stabilizing term is proportional to the mismatch produced by violating the compatibility condition. We show that the modified method is consistent with the water wave equations, and it has the same order of accuracy as the point vortex method. Moreover, the added stabilizing term does not introduce new destabilizing terms. This stabilizing technique seems to be quite general. It can be generalized to nonperiodic or 3-D fluid surfaces.

The organization of the rest of the paper is as follows. In Section 2 we present the boundary integral method for the 2-D water wave problem. The compatibility conditions are discussed there. We introduce the new stabilizing technique in Section 3 and present the stability analysis. Some numerical results are presented in Section 4 to illustrate the effect of various stabilizing terms.

\section{BOUNDARY INTEGRAL FORMULATION AND DISCRETIZATION}

Consider the two-dimensional incompressible, inviscid and irrotational fluid flow separated by a free interface. We assume the fluid above the interface is air with zero density, and the fluid below the interface has infinite depth. We represent in 
parametric form the position of the water interface by a complex variable, $z(\alpha, t)$. The parameter $\alpha$ is the Lagrangian variable. Furthermore, we denote by $\phi(\alpha, t)$ the velocity potential on the interface, and the real and imaginary parts of $z$ as $x$ and $y$, i.e., $z(\alpha, t)=x(\alpha, t)+i y(\alpha, t)$. To express the evolution of the interface, we need to write the velocity on the surface in terms of these variables. Following [1], we begin with a double layer or dipole representation for the potential in terms of the dipole strength $\mu(\alpha)$, to be determined from $\phi(\alpha)$. We write the complex potential in the fluid domain as

$$
\Phi(z)=\frac{1}{2 \pi i} \int \frac{\mu\left(\alpha^{\prime}\right)}{z-z\left(\alpha^{\prime}\right)} d z\left(\alpha^{\prime}\right) .
$$

The real potential in the interior is

$$
\phi(z)=\operatorname{Re} \Phi(z)=\int \frac{\partial}{\partial n\left(\alpha^{\prime}\right)} G\left(z-z\left(\alpha^{\prime}\right)\right) \mu\left(\alpha^{\prime}\right) d s\left(\alpha^{\prime}\right),
$$

where $G(z)=(2 \pi)^{-1} \log |z|$ is the free space Green function for the 2-D Laplace equation, $\frac{\partial}{\partial n\left(\alpha^{\prime}\right)}$ is the normal derivative along the interface. For simplicity, we often drop the time variable from now on, but all the quantities, $z, \mu, \phi$ will be time dependent. It follows from the properties of the double layer potential that the value of $\phi$ on the interface is given by

$$
\phi(\alpha)=\frac{1}{2} \mu(\alpha)+\operatorname{Re}\left\{\frac{1}{2 \pi i} \int \frac{\mu\left(\alpha^{\prime}\right)}{z(\alpha)-z\left(\alpha^{\prime}\right)} d z\left(\alpha^{\prime}\right)\right\},
$$

where $\phi(\alpha)=\phi(z(\alpha))$, and the integral is understood as the Cauchy principle value integral. Differentiating equation (2) with respect to $\alpha$ and integrating by parts we obtain

$$
\phi_{\alpha}(\alpha)=\frac{\gamma(\alpha)}{2}+\operatorname{Re}\left[\frac{z_{\alpha}(\alpha)}{2 \pi i} \int \frac{\gamma\left(\alpha^{\prime}\right)}{z(\alpha)-z\left(\alpha^{\prime}\right)} d \alpha^{\prime}\right],
$$

which defines an integral equation for $\gamma$. Here $\gamma=\mu_{\alpha}$ is the nonnormalized vortex sheet strength. The complex velocity $w=u-i v$ can be obtained by differentiating the complex potential with respect to $z$. We get

$$
w(z)=\frac{d \Phi}{d z}(z)=\frac{1}{2 \pi i} \int \frac{\gamma\left(\alpha^{\prime}\right)}{z-z\left(\alpha^{\prime}, t\right)} d \alpha^{\prime} .
$$

Using the Plemelj formula (see, e.g., [9], p. 316), we obtain the fluid velocity on the interface

$$
w(\alpha)=\frac{1}{2 \pi i} \int \frac{\gamma\left(\alpha^{\prime}\right)}{z(\alpha)-z\left(\alpha^{\prime}\right)} d \alpha^{\prime}+\frac{\gamma(\alpha)}{2 z_{\alpha}(\alpha)},
$$

where we have taken the limit of $z$ approaching the free surface $z(\alpha)$ from the fluid region and $w(\alpha)=w(z(\alpha))$. The integral is understood as the Cauchy principle value integral. Since $\alpha$ is the Lagrangian variable, the velocity on the interface is that of the fluid below. We obtain an evolution equation for the interface 3 .

$$
\frac{\partial \bar{z}}{\partial t}(\alpha)=w(\alpha)
$$

where $\bar{z}$ is the complex conjugate of $z$. For the evolution of $\phi(\alpha)$, we use the Bernoulli equation. If we neglect surface tension, the Bernoulli equation in the Lagrangian frame is 3 .

$$
\phi_{t}-\frac{1}{2}|w|^{2}+g y=0
$$


where $g$ is the gravity coefficient. The evolution equations (6) and (7) together with the relations (3) and (5) completely specify the motion of the system.

From now on, with $z(\alpha, t)=\alpha+s(\alpha, t)$, we assume that $s(\alpha, t)$ and $\phi(\alpha, t)$ are periodic in $\alpha$ with period $2 \pi$. To reduce the computational domain to a single period, we need to replace the original Green's function by a periodic one, which is obtained by summing up all its periodic images. The singular kernel $\frac{1}{z}$ in the velocity integrand becomes $\frac{1}{2} \cot \left(\frac{z}{2}\right)$ (this can be derived following a similar argument leading to equation (26) in Section 3). To summarize, we obtain a system of time evolution equations for $z$ and $\phi$ as follows:

$$
\begin{aligned}
\frac{d \bar{z}}{d t}(\alpha) & =\frac{1}{4 \pi i} \int_{0}^{2 \pi} \gamma\left(\alpha^{\prime}\right) \cot \left(\frac{z(\alpha)-z\left(\alpha^{\prime}\right)}{2}\right) d \alpha^{\prime}+\frac{\gamma(\alpha)}{2 z_{\alpha}(\alpha)} \\
& \equiv u(\alpha)-i v(\alpha) \\
\phi_{t}(\alpha) & =\frac{1}{2}\left(u^{2}+v^{2}\right)(\alpha)-g y(\alpha) \\
\phi_{\alpha}(\alpha) & =\frac{\gamma(\alpha)}{2}+\operatorname{Re}\left[\frac{z_{\alpha}(\alpha)}{4 \pi i} \int_{0}^{2 \pi} \gamma\left(\alpha^{\prime}\right) \cot \left(\frac{z(\alpha)-z\left(\alpha^{\prime}\right)}{2}\right) d \alpha^{\prime}\right] .
\end{aligned}
$$

Equations (8)- (10) completely determine the motion of the system. A unique solution is specified by giving initial conditions for the interface position $z$ and velocity potential $\phi$. It can be shown that the integral equation (10) for the vortex sheet strength $\gamma$ can be solved in terms of $\phi_{\alpha}$ [1] (note that the integral kernel for $\gamma$ is an adjoint operator of the integral kernel for $\mu$ ). This is done at each time step. We then use the interface equation (8) and the Bernoulli equation (9) to update $z$ and $\phi$, respectively.

In order to discretize the water wave equations (8)-(10), we need to make choices for a discrete derivative operator and a quadrature rule. We approximate the velocity integral by the point vortex method [11. To describe the discrete derivative operator, we recall that the discrete Fourier transform is defined by

$$
\hat{u}_{k}=\frac{1}{N} \sum_{j=1}^{N} u\left(\alpha_{j}\right) e^{-i k \alpha_{j}}, \quad \alpha_{j}=j h, \quad h=\frac{2 \pi}{N}, \quad k=-N / 2+1, \ldots, N / 2 .
$$

The inversion formula reads

$$
u\left(\alpha_{j}\right)=\sum_{k=-N / 2+1}^{N / 2} \hat{u}_{k} e^{i k \alpha_{j}} .
$$

Here we assume that $N$ is even. We note that $\hat{u}_{k}$ is periodic in $k$ with period $N$. A discrete derivative operator, denoted as $D_{h}$, may be expressed in the Fourier transform as follows

$$
{\widehat{\left(D_{h} f\right.}}_{k}=i k \rho(k h) \hat{f}_{k}, \quad k=-\frac{N}{2}+1, \ldots, \frac{N}{2} .
$$

We assume that $\rho(k h) \geq 0$. The choice of $\rho$ depends on the discrete derivative operator. For the second-order centered difference operator, we have

$$
\rho_{2}(k h)=\frac{\sin (k h)}{k h} \geq 0 ;
$$

for the fourth-order centered differencing,

$$
\rho_{4}(k h)=\frac{8 \sin (k h)-\sin (2 k h)}{6 k h} \geq 0 ;
$$


for the cubic spline approximation,

$$
\rho_{c}(k h)=\frac{\sin (k h)}{k h}\left(\frac{3}{2+\cos (k h)}\right) \geq 0 .
$$

For the pseudo-spectral derivative with smoothing, $\rho(k h)$ satisfies the following properties: (i) $\rho(x)$ is a nonnegative even function; (ii) $\rho(\cdot) \in C^{2}$ and $\rho(\pi)=0$; and (iii) $\rho(x)=1$ for $|x| \leq \lambda \pi$ where $0<\lambda<1$. Property (iii) guarantees that $D_{h}$ is spectrally accurate.

Denote by $z_{j}(t)$ the discrete approximation of $z\left(\alpha_{j}, t\right)$, where $\alpha_{j}=j h, j=$ $1,2, \ldots, N, h=2 \pi / N$ is the mesh size, and $N$ is the number of grid points in one periodic interval, $[0,2 \pi]$. The quantities $\phi_{j}(t)$ and $\gamma_{j}(t)$ are defined similarly. Then the discrete complex interface velocity, denoted as $w_{j}$, is given by

$$
w_{j}=u_{j}-i v_{j}=\frac{1}{4 \pi i} \sum_{k=1, k \neq j}^{N} \gamma_{k} \cot \left(\frac{z_{j}-z_{k}}{2}\right) h+\frac{\gamma_{j}}{2 D_{h} z_{j}} .
$$

Now we can present the point vortex method for the water wave equations (8)-(10) as follows:

$$
\begin{aligned}
\frac{d}{d t} \bar{z}_{j} & =\frac{1}{4 \pi i} \sum_{k=1, k \neq j}^{N} \gamma_{k} \cot \left(\frac{z_{j}-z_{k}}{2}\right) h+\frac{\gamma_{j}}{2 D_{h} z_{j}} \equiv u_{j}-i v_{j} \\
\frac{d \phi_{j}}{d t} & =\frac{1}{2}\left(u_{j}^{2}+v_{j}^{2}\right)-g y_{j} \\
D_{h} \phi_{j} & =\frac{1}{2} \gamma_{j}+\operatorname{Re}\left[\frac{D_{h} z_{j}}{4 \pi i} \sum_{k=1, k \neq j}^{N} \gamma_{k} \cot \left(\frac{z_{j}-z_{k}}{2}\right) h\right] .
\end{aligned}
$$

These equations can be solved once initial conditions are specified for $z_{j}$ and $\phi_{j}$ and a time discretization is chosen. The integral equation (14) must be solved for $\gamma_{j}$ at each time step. In practice it is solved iteratively (see Section 4).

There are many ways by which one can discretize the singular integral and the derivative. These choices affect critically the accuracy and stability of the numerical method. Straightforward numerical discretizations, such as (12)-(14), may lead to rapid growth of the numerical solution in the high wavenumbers. To avoid numerical instability, a certain compatibility between the choice of the quadrature rule for the singular integral and the discrete derivative must be satisfied. This compatibility ensures that a delicate balance of terms that hold on the continuous level is preserved on the discrete level. Violation of this compatibility will lead to numerical instability [3]. The compatibility condition mentioned above is related to the properties of the continuous Hilbert transform. Recall that the Hilbert transform is defined as follows:

$$
H(f)(\alpha)=\frac{1}{2 \pi} \int_{0}^{2 \pi} \cot \left(\frac{\alpha-\alpha^{\prime}}{2}\right) f\left(\alpha^{\prime}\right) d \alpha^{\prime}, \quad \text { for } \quad f \in L^{2}[0,2 \pi] .
$$

A related $\Lambda$ operator is defined as

$$
\Lambda(f)(\alpha)=\frac{1}{4 \pi} \int_{0}^{2 \pi} \frac{\left(f(\alpha)-f\left(\alpha^{\prime}\right)\right) d \alpha^{\prime}}{\sin ^{2}\left(\alpha-\alpha^{\prime}\right)}, \quad \text { for } \quad f \in L^{2}[0,2 \pi] .
$$


It is easy to show that the $\Lambda$ operator and the Hilbert transform satisfy the properties

$$
\Lambda=H D_{\alpha}, \quad H^{2} D_{\alpha}=-D_{\alpha}
$$

where $D_{\alpha}$ is a derivative operator. These two properties are essential in obtaining the well-posedness of the water wave equations [14. Linear stability analysis away from equilibrium seems to indicate that the corresponding discrete operators need to satisfy similar relations for stability [3. More specifically, if we define the discrete Hilbert transform, $H_{h}$, and the discrete $\Lambda$ operator, $\Lambda_{h}$, as follows

$$
\begin{aligned}
& H_{h}\left(f_{j}\right)=\frac{1}{2 \pi} \sum_{k=1, k \neq j}^{N} \cot \left(\frac{\alpha_{j}-\alpha_{k}}{2}\right) f_{k} h, \\
& \Lambda_{h}\left(f_{j}\right)=\frac{1}{4 \pi} \sum_{k=1, k \neq j}^{N} \frac{\left(f_{j}-f_{k}\right)}{\sin ^{2}\left(\alpha_{j}-\alpha_{k}\right)} h,
\end{aligned}
$$

then the discrete compatibility condition is given by

$$
\begin{aligned}
& \Lambda_{h}\left(\dot{z}_{j}\right)=H_{h} D_{h}\left(\dot{z}_{j}\right), \\
& \left(H_{h}^{2}+I\right) D_{h}\left(\dot{z}_{j}\right)=0 .
\end{aligned}
$$

In [3], Beale et al. use the alternating trapezoidal rule to approximate the velocity integral, which gives a spectrally accurate approximation. In this case, property (17) is automatically satisfied. Fourier filtering on the interface variable, $z_{j}$, can be used to enforce the compatibility condition (16). For a periodic perturbation of the flat interface, $z(\alpha, t)=\alpha+s(\alpha, t)$, the Fourier filtering on $z$ is defined as $z_{j}^{p}=\alpha+s_{j}^{p}$, where $\hat{s}_{k}^{p}=p(k h) \hat{s}_{k}, p$ is a nonnegative cut-off function in the frequency domain, and $\hat{s}_{k}$ is the discrete Fourier transform of $s$ with wavenumber $k$. The cut-off function $p$ is determined by satisfying a modified compatibility condition $\Lambda_{h}\left(\dot{z}_{j}^{p}\right)=H_{h} D_{h}\left(\dot{z}_{j}\right)$. We refer to Section 4 for more discussions.

On the other hand, for the point vortex method approximation which is only first order accurate, it seems very difficult, if not impossible, to use the Fourier filtering to enforce both compatibility conditions (16) and (17). This is also the essential difficulty for 3-D free surface problems since there seems to be no spectrally accurate discretization available for 3 -D interfaces due to the branch point singularity in the 3-D Green's function. In the next section, we will introduce a new stability technique to enforce both compatibility conditions indirectly. This technique gives a stable discretization of the point vortex method for 2-D water waves.

\section{A NeW Stabilizing technique for 2-D Water WaVes}

In this section, we will illustrate the main idea of our new stabilizing technique for the point vortex method for 2-D water waves. Let $w_{j}$ be the point vortex method approximation to the complex interface velocity, $w\left(\alpha_{j}\right)$ (see (11)). We modify the 
point vortex method as follows:

$$
\begin{aligned}
\frac{d \bar{z}_{j}}{d t} & =w_{j}+A_{j}+B_{j} \equiv u_{j}-i v_{j} \\
\frac{d \phi_{j}}{d t} & =\frac{1}{2}\left(u_{j}^{2}+v_{j}^{2}\right)-g y_{j} \\
D_{h} \phi_{j} & =\frac{\gamma_{j}}{2}+\operatorname{Re}\left(D_{h} z_{j}\left(\frac{1}{4 \pi i} \sum_{k=1, k \neq j}^{N} \gamma_{k} \cot \left(\frac{z_{j}-z_{k}}{2}\right) h+A_{j}\right)\right)
\end{aligned}
$$

where $A$ and $B$ are our stabilizing terms defined as

$$
\begin{aligned}
A_{j} & =\frac{\gamma_{j}}{2 i\left(D_{h} z_{j}\right)^{2}}\left(\Lambda_{h}-H_{h} D_{h}\right) z_{j} \\
B_{j} & =-\frac{1}{D_{h} z_{j}}\left(\left(I+H_{h}^{2}\right) D_{h} \phi_{j}-\operatorname{Re}\left(w_{j}\left(I+H_{h}^{2}\right) D_{h} z_{j}\right)\right) \\
& -\frac{1}{2 i D_{h} z_{j}} \operatorname{Im}\left(\frac{\gamma_{j}}{D_{h} z_{j}}\left(I+H_{h}^{2}\right) D_{h} z_{j}\right) .
\end{aligned}
$$

The first stabilizing term, $A$, is to enforce the compatibility condition of $\Lambda_{h}=$ $H_{h} D_{h}$, while the second stabilizing term, $B$, is to eliminate the destabilizing terms introduced by violating the compatibility condition, $H_{h}^{2} D_{h}=-D_{h}$. The role of $A$ and $B$ in stabilizing the point vortex method will become clear as we perform stability analysis far from equilibrium. Note that both stabilizing terms are discrete convolution operators. Hence they can be evaluated in $O(N \log (N))$ operations by the Fast Fourier Transform.

The main result of this section is the following convergence result.

Theorem 1. Assume that the water wave problem is well-posed and has a smooth solution in $C^{m+2}(m \geq 3)$ up to time $T$. Let $D_{h}$ be a r-th order derivative approximation with Fourier symbol $\rho$, i.e., $\widehat{\left(D_{h}\right)_{k}}=i k \rho(k h)$. We further assume that $r \geq 2, \rho(x) \geq 0$, and $\rho(\pi)=0$. Then the modified point vortex method (18) - 22) is stable and convergent. More precisely, there exist positive constants, $h_{0}(T)$ and $C(T)$ independent of $h$ such that for $0<h \leq h_{0}(T)$ we have

$$
\|z(t)-z(\cdot, t)\|_{l^{2}} \leq C(T) h .
$$

Similar convergent results hold for $\phi_{j}$ and $\gamma_{j}$. Here $\|z\|_{l^{2}}^{2}=\sum_{j=1}^{N}\left|z_{j}\right|^{2} h$.

Proof of Theorem 1. First of all, we note that the modified algorithm is consistent with the water wave equations since the point vortex method is a first-order approximation to the singular velocity integral 4]. For a smooth interface solution, $z(\alpha)$, we have

$$
\left(\Lambda_{h}-H_{h} D_{h}\right) z\left(\alpha_{j}\right)=O(h), \quad\left(I+H_{h}^{2}\right) z_{\alpha}\left(\alpha_{j}\right)=O(h),
$$

where we have used the compatibility conditions at the continuous level, i.e., $\Lambda=$ $H D$ and $H^{2} D=-D$. Thus, the modified point vortex method gives a first order approximation to the 2-D water wave equations. In fact, using the same argument as in [6], we can prove that the numerical error in the point vortex method approximation has a power series expansion in the odd powers of $h$

$$
w_{j}=w\left(\alpha_{j}\right)+C_{1}\left(\alpha_{j}\right) h+C_{3}\left(\alpha_{j}\right) h^{3}+C_{5}\left(\alpha_{j}\right) h^{5}+\cdots .
$$


Similar error expansions also hold for the correction terms, $A$ and $B$. The existence of the error expansion enables us to use Strang's argument to obtain nonlinear stability and convergence (see [12]).

Denote the errors in $z_{j}, \gamma_{j}$ and $\phi_{j}$ by $\dot{z}_{j}=z_{j}-z\left(\alpha_{j}\right), \dot{\gamma}_{j}=\gamma_{j}-\gamma\left(\alpha_{j}\right)$, and $\dot{\phi}_{j}=\phi_{j}-\phi\left(\alpha_{j}\right)$, respectively. Define

$$
E_{j}=\frac{1}{4 \pi i} \sum_{k=1, k \neq j}^{N} \gamma_{k} \cot \left(\frac{z_{j}-z_{k}}{2}\right) h \quad \text { and } \quad \zeta_{j}=\frac{\gamma_{j}}{D_{h} z_{j}}
$$

Accordingly, we define $\dot{E}_{j}=E_{j}-E\left(\alpha_{j}\right), \dot{A}_{j}=A_{j}-A\left(\alpha_{j}\right), \dot{B}_{j}=B_{j}-B\left(\alpha_{j}\right)$, and $\dot{\zeta}_{j}=\zeta_{j}-\zeta\left(\alpha_{j}\right)$. Here the quantities, $E\left(\alpha_{j}\right), A\left(\alpha_{j}\right)$, and $B\left(\alpha_{j}\right)$ are defined by replacing $z_{j}$ by $z\left(\alpha_{j}\right)$ and $\gamma_{j}$ by $\gamma\left(\alpha_{j}\right)$, in (25), (21) and (22). To prove stability, we need to show that $\dot{z}, \dot{\phi}$ and $\dot{\gamma}$ are bounded in a suitable norm for as long as a smooth solution exists. To achieve this, we need to estimate $\dot{E}_{j}, \dot{\zeta}_{j}, \dot{A}_{j}$ and $\dot{B}_{j}$, etc., in terms of $\dot{z}_{j}, \dot{\phi}_{j}$, and $\dot{\gamma}_{j}$. It is sufficient to consider the linear stability around an arbitrary smooth solution. The nonlinear stability can be obtained in a manner similar to that used in [3] for a spectrally accurate discretization by using Strang's argument [12]. We first study the linear variation of $E_{j}$. For technical reasons, it is easier to work with the kernel in the infinite domain than in the periodic domain. For this purpose, we first extend the sum over a single period to the sum over the whole line. Note that

$$
\frac{1}{2} \cot \left(\frac{z}{2}\right)=\frac{1}{z}+\sum_{m=1}^{\infty} \frac{2 z}{z^{2}-(2 m \pi)^{2}}
$$

which converges absolutely away from $z=2 m \pi$ for any integer $m$. Thus we have

$$
\begin{aligned}
& \frac{1}{2} \sum_{k=1, k \neq j}^{N} \gamma\left(\alpha_{k}\right) \cot \left(\frac{z\left(\alpha_{j}\right)-z\left(\alpha_{k}\right)}{2}\right) h \\
& \quad=\sum_{k=1, k \neq j}^{N} \frac{\gamma\left(\alpha_{k}\right) h}{z\left(\alpha_{j}\right)-z\left(\alpha_{k}\right)}+\sum_{m=1}^{\infty} \sum_{k=1, k \neq j}^{N} \frac{2\left(z\left(\alpha_{j}\right)-z\left(\alpha_{k}\right)\right) \gamma\left(\alpha_{k}\right) h}{\left(z\left(\alpha_{j}\right)-z\left(\alpha_{k}\right)\right)^{2}-(2 m \pi)^{2}}
\end{aligned}
$$

Recall that $z(\alpha)=\alpha+s(\alpha), s(\alpha)$ and $\gamma(\alpha)$ are $2 \pi$-periodic. Define $\alpha_{j+m N}=$ $\alpha_{j}+2 m \pi$. We obtain after some algebra that

$$
\begin{aligned}
\sum_{m=1}^{M} & \sum_{k=1, k \neq j}^{N} \frac{2\left(z\left(\alpha_{j}\right)-z\left(\alpha_{k}\right)\right) \gamma\left(\alpha_{k}\right) h}{\left.z\left(\alpha_{j}\right)-z\left(\alpha_{k}\right)\right)^{2}-(2 m \pi)^{2}} \\
& =\sum_{m=1}^{M} \sum_{k=1, k \neq j}^{N}\left(\frac{\gamma\left(\alpha_{k}\right)}{z\left(\alpha_{j}\right)-z\left(\alpha_{k}\right)-2 m \pi}+\frac{\gamma\left(\alpha_{k}\right)}{z\left(\alpha_{j}\right)-z\left(\alpha_{k}\right)+2 m \pi}\right) h \\
& =\sum_{m=1}^{M} \sum_{k=1, k \neq j}^{N}\left(\frac{\gamma\left(\alpha_{k+m N}\right)}{z\left(\alpha_{j}\right)-z\left(\alpha_{k+m N}\right)}+\frac{\gamma\left(\alpha_{k-m N}\right)}{z\left(\alpha_{j}\right)-z\left(\alpha_{k-m N}\right)}\right) h \\
& =\sum_{k=-M N+1, k \neq j}^{(M+1) N}\left(\frac{\gamma\left(\alpha_{k}\right)}{z\left(\alpha_{j}\right)-z\left(\alpha_{k}\right)}\right) h-\sum_{k=1, k \neq j}^{N}\left(\frac{\gamma\left(\alpha_{k}\right)}{z\left(\alpha_{j}\right)-z\left(\alpha_{k}\right)}\right) h .
\end{aligned}
$$


Thus, we get

$$
\begin{aligned}
\frac{1}{2} \sum_{k=1, k \neq j}^{N} \gamma\left(\alpha_{k}\right) & \cot \left(\frac{z\left(\alpha_{j}\right)-z\left(\alpha_{k}\right)}{2}\right) h \\
& =\lim _{M \rightarrow \infty} \sum_{k=-M N+1, k \neq j}^{(M+1) N}\left(\frac{\gamma\left(\alpha_{k}\right)}{z\left(\alpha_{j}\right)-z\left(\alpha_{k}\right)}\right) h .
\end{aligned}
$$

In the rest of the paper, we will use the notation

$$
\sum_{k \neq j} \frac{\gamma\left(\alpha_{k}\right) h}{z\left(\alpha_{j}\right)-z\left(\alpha_{k}\right)} \equiv \lim _{M \rightarrow \infty} \sum_{k=-M N+1, k \neq j}^{(M+1) N}\left(\frac{\gamma\left(\alpha_{k}\right)}{z\left(\alpha_{j}\right)-z\left(\alpha_{k}\right)}\right) h .
$$

Therefore, we have

$$
E_{j}=\frac{1}{2 \pi i} \sum_{k \neq j} \frac{\gamma_{k} h}{z_{j}-z_{k}}
$$

Direct calculations show that the linear variation $\dot{E}_{j}^{L}$ is given by

$$
\dot{E}_{j}^{L} \equiv \frac{1}{2 \pi i} \sum_{k \neq j} \frac{\dot{\gamma}_{k}}{z\left(\alpha_{j}\right)-z\left(\alpha_{k}\right)} h-\frac{1}{2 \pi i} \sum_{k \neq j} \frac{\gamma\left(\alpha_{k}\right)\left(\dot{z}_{j}-\dot{z}_{k}\right)}{\left(z\left(\alpha_{j}\right)-z\left(\alpha_{k}\right)\right)^{2}} h
$$

Note that

$$
\frac{1}{z\left(\alpha_{j}\right)-z\left(\alpha_{k}\right)}=\frac{1}{z_{\alpha}\left(\alpha_{j}\right)\left(\alpha_{j}-\alpha_{k}\right)}+f\left(\alpha_{j}, \alpha_{k}\right)
$$

for some smooth function $f(\alpha, \beta)$. Thus, to the leading order, $\dot{E}_{j}^{L}$ becomes

$$
\dot{E}_{j}^{L}=\frac{1}{2 i z_{\alpha}\left(\alpha_{j}\right)} H_{h}\left(\dot{\gamma}_{j}\right)-\frac{\gamma\left(\alpha_{j}\right)}{2 i z_{\alpha}\left(\alpha_{j}\right)^{2}} \Lambda_{h} \dot{z}_{j}+A_{-1}\left(\dot{\gamma}_{j}\right)+A_{0}\left(\dot{z}_{j}\right)
$$

where $A_{0}$ is a bounded operator from $l^{2}$ to $l^{2}$, and $A_{-1}$ is a discrete smoothing operator of order one, i.e., $D_{h} A_{-1}=A_{0}$ and $A_{-1} D_{h}=A_{0}$. Here we have used the fact that the commutator $\left[H_{h}, g\right]=H_{h} g-g H_{h}=A_{-1}$ is a smoothing operator for smooth $g$, and $\left[\Lambda_{h}, g\right]=A_{0}$. This is not true for the alternating trapezoidal rule due to aliasing errors.

Next we estimate the stability error for our first correction term:

$$
\begin{aligned}
\dot{A}_{j}^{L}= & \left(\frac{\gamma_{j}}{2 i\left(D_{h} z_{j}\right)^{2}}-\frac{\gamma\left(\alpha_{j}\right)}{2 i D_{h} z\left(\alpha_{j}\right)}\right)\left(\Lambda_{h}-H_{h} D_{h}\right) z\left(\alpha_{j}\right) \\
& +\frac{\gamma\left(\alpha_{j}\right)}{2 i z_{\alpha}^{2}\left(\alpha_{j}\right)}\left(\Lambda_{h}-H_{h} D_{h}\right) \dot{z}_{j} .
\end{aligned}
$$

Observe that for a smooth solution $z(\alpha)$, we have $\left(\Lambda_{h}-H_{h} D_{h}\right) z\left(\alpha_{j}\right)=O(h)$. Therefore, we have to the leading order that

$$
\dot{A}_{j}^{L}=\frac{\gamma\left(\alpha_{j}\right)}{2 i z_{\alpha}^{2}\left(\alpha_{j}\right)}\left(\Lambda_{h}-H_{h} D_{h}\right) \dot{z}_{j}+A_{0}\left(\dot{z}_{j}\right)+A_{-1}\left(\dot{\gamma}_{j}\right) .
$$


Now combining $\dot{E}_{j}^{L}$ with $\dot{A}^{L}$, we obtain

$$
\begin{aligned}
\dot{E}_{j}^{L}+\dot{A}_{j}^{L}= & \frac{1}{2 i z_{\alpha}\left(\alpha_{j}\right)} H_{h}\left(\dot{\gamma}_{j}\right)-\frac{\gamma\left(\alpha_{j}\right)}{2 i z_{\alpha}\left(\alpha_{j}\right)^{2}} \Lambda_{h} \dot{z}_{j} \\
& +\frac{\gamma\left(\alpha_{j}\right)}{2 i z_{\alpha}^{2}\left(\alpha_{j}\right)}\left(\Lambda_{h}-H_{h} D_{h}\right) \dot{z}_{j}+A_{0}\left(\dot{z}_{j}\right)+A_{-1}\left(\dot{\gamma}_{j}\right) \\
= & \frac{1}{2 i z_{\alpha}\left(\alpha_{j}\right)} H_{h}\left(\dot{\gamma}_{j}\right)-\frac{\gamma\left(\alpha_{j}\right)}{2 i z_{\alpha}^{2}\left(\alpha_{j}\right)} H_{h} D_{h} \dot{z}_{j}+A_{0}\left(\dot{z}_{j}\right)+A_{-1}\left(\dot{\gamma}_{j}\right)
\end{aligned}
$$

Note that the two $\Lambda_{h} \dot{z}_{j}$ terms cancel each other in the above equation, and only the $H_{h} D_{h} \dot{z}_{j}$ term survives in place of $\Lambda_{h} \dot{z}_{j}$. Thus, in effect we enforce the compatibility condition $\Lambda_{h}=H_{h} D_{h}$ by adding the stabilizing term $A$. It is important that the coefficient $\frac{\gamma_{j}}{2 i\left(D_{h} z_{j}\right)^{2}}$ in front of $\left(\Lambda_{h}-H_{h} D_{h}\right) z_{j}$ does not give rise to any new destabilizing term to the leading order because $\left(\Lambda_{h}-H_{h} D_{h}\right) z\left(\alpha_{j}\right)=O(h)$ is small. This is why the we can add a stabilizing term $A$ to exactly cancel the destabilizing term corresponding to the violation of the compatibility condition $\Lambda_{h}=H_{h} D_{h}$.

To summarize, we have

$$
\begin{aligned}
\dot{E}_{j}^{L}+\dot{A}_{j}^{L}+\dot{\zeta}_{j}^{L}= & \frac{1}{2 z_{\alpha}\left(\alpha_{j}\right)}\left(I-i H_{h}\right)\left(\dot{\gamma}_{j}-\frac{\gamma\left(\alpha_{j}\right)}{z_{\alpha}\left(\alpha_{j}\right)} D_{h} \dot{z}_{j}\right) \\
& +A_{0}\left(\dot{z}_{j}\right)+A_{-1}\left(\dot{\gamma}_{j}\right) .
\end{aligned}
$$

Using the estimate for $\dot{E}_{j}+\dot{A}_{j}$, we can also obtain an estimate for $\dot{\gamma}_{j}$ following an argument similar to that in 3 ]

$$
\begin{aligned}
\dot{\gamma}_{j}= & 2 D_{h} \dot{\phi}_{j}-2 \operatorname{Re}\left(w_{0}\left(\alpha_{j}\right) D_{h} \dot{z}_{j}\right)+D_{h} H_{h} \operatorname{Im}\left(\frac{\gamma\left(\alpha_{j}\right)}{z_{\alpha}\left(\alpha_{j}\right)} \dot{z}_{j}\right) \\
& +A_{0}\left(\dot{z}_{j}\right)+A_{0}\left(\dot{\phi}_{j}\right)
\end{aligned}
$$

where $w_{0}$ is the integral part of the interface velocity, i.e., $w=w_{0}+\zeta$.

Next we will consider the role of our second correction term, $B$, which is used to stabilize the terms introduced by violating the second compatibility condition, $\left(I+H_{h}^{2}\right) D_{h}=0$. Using the identity

$$
\left(I-i H_{h}\right)\left(I+i H_{h}\right) D_{h}=\left(I+H_{h}^{2}\right) D_{h}
$$

we get

$$
\left(I-i H_{h}\right) H_{h} D_{h}=\left(I-i H_{h}\right)\left(i D_{h}\right)+\frac{1}{i}\left(I+H_{h}^{2}\right) D_{h} .
$$

Substituting (34) into (33) and using the above identity, we obtain by following the same argument as in [3] that

$$
\begin{aligned}
\frac{d \bar{z}_{j}}{d t}= & \frac{1}{z_{\alpha}\left(\alpha_{j}\right)}\left(I-i H_{h}\right) D_{h}\left[\dot{\phi}_{j}-\operatorname{Re}\left(w\left(\alpha_{j}\right) \dot{z}_{j}\right)\right] \\
& +\frac{1}{2 i z_{\alpha}\left(\alpha_{j}\right)}\left(I+H_{h}^{2}\right) \operatorname{Im}\left(\frac{\gamma\left(\alpha_{j}\right) D_{h} \dot{z}_{j}}{z_{\alpha}\left(\alpha_{j}\right)}\right)+\dot{B}_{j}+A_{0}\left(\dot{z}_{j}\right)+A_{0}\left(\dot{\phi}_{j}\right) .
\end{aligned}
$$


We now estimate the linear variation of the second stabilizing term, $\dot{B}$. Recall that

$$
\begin{aligned}
B_{j}=-\frac{1}{D_{h} z_{j}}((I & \left.\left.+H_{h}^{2}\right) D_{h} \phi_{j}-\operatorname{Re}\left(w_{j}\left(I+H_{h}^{2}\right) D_{h} z_{j}\right)\right) \\
& -\frac{1}{2 i D_{h} z_{j}} \operatorname{Im}\left(\frac{\gamma_{j}}{D_{h} z_{j}}\left(I+H_{h}^{2}\right) D_{h} z_{j}\right) .
\end{aligned}
$$

By a direct calculation, one can show that

$$
\begin{aligned}
\dot{B}_{j}^{L}= & -\frac{1}{z_{\alpha}\left(\alpha_{j}\right)}\left(\left(I+H_{h}^{2}\right) D_{h} \dot{\phi}_{j}-\operatorname{Re}\left(w\left(\alpha_{j}\right)\left(I+H_{h}^{2}\right) D_{h} \dot{z}_{j}\right)\right) \\
& -\frac{1}{2 i z_{\alpha}\left(\alpha_{j}\right)} \operatorname{Im}\left(\frac{\gamma\left(\alpha_{j}\right)}{z_{\alpha}\left(\alpha_{j}\right)}\left(I+H_{h}^{2}\right) D_{h} \dot{z}_{j}\right)+A_{0}\left(\dot{z}_{j}\right)+A_{-1}\left(\dot{\gamma}_{j}\right),
\end{aligned}
$$

where we have used the fact that $\left(I+H_{h}^{2}\right) z_{\alpha}\left(\alpha_{j}\right)=O(h)$. Define

$$
\dot{F}_{j}=\dot{\phi}_{j}-\operatorname{Re}\left(w\left(\alpha_{j}\right) \dot{z}_{j}\right) \text {. }
$$

Using the fact that the commutator $\left[\left(I+H_{h}^{2}\right), g\right]=A_{-1}$ for smooth $g$, we have

$$
\begin{aligned}
\dot{B}_{j}^{L}= & -\frac{1}{z_{\alpha}\left(\alpha_{j}\right)}\left(I+H_{h}^{2}\right) D_{h} \dot{F}_{j}-\frac{1}{2 i z_{\alpha}\left(\alpha_{j}\right)} \operatorname{Im}\left(\frac{\gamma\left(\alpha_{j}\right)}{z_{\alpha}\left(\alpha_{j}\right)}\left(I+H_{h}^{2}\right) D_{h} \dot{z}_{j}\right) \\
& +A_{0}\left(\dot{z}_{j}\right)+A_{-1}\left(\dot{\gamma}_{j}\right) .
\end{aligned}
$$

Substituting the estimate for $\dot{B}_{j}^{L}$ into (35), we get after some cancellations that

$$
\begin{aligned}
\frac{d \bar{z}_{j}}{d t}= & \frac{1}{z_{\alpha}\left(\alpha_{j}\right)}\left(I-i H_{h}\right) D_{h} \dot{F}_{j}-\frac{1}{z_{\alpha}\left(\alpha_{j}\right)}\left(I+H_{h}^{2}\right) D_{h} \dot{F}_{j} \\
& +A_{0}\left(\dot{z}_{j}\right)+A_{0}\left(\dot{\phi}_{j}\right) .
\end{aligned}
$$

In order to complete our energy estimate, it is important to project the errors into local normal and tangent vectors

$$
\dot{z}^{T}=(\dot{x}, \dot{y}) \cdot \mathbf{t}, \quad \dot{z}^{N}=(\dot{x}, \dot{y}) \cdot \mathbf{n},
$$

where $\mathbf{t}=\sigma(\alpha)\left(x_{\alpha}, y_{\alpha}\right), \mathbf{n}=\sigma(\alpha)\left(-y_{\alpha}, x_{\alpha}\right)$ are the unit tangent and normal vectors, respectively, $\sigma=\left(x_{\alpha}^{2}+y_{\alpha}^{2}\right)^{-1 / 2}$. After projecting the error equation (38) into the local tangent and normal vectors, we obtain

$$
\begin{aligned}
& \left(\dot{z}_{j}^{T}\right)_{t}=\sigma\left(\alpha_{j}\right) D_{h} \dot{F}_{j}-\sigma\left(\alpha_{j}\right)\left(I+H_{h}^{2}\right) D_{h} \dot{F}_{j}+A_{0}\left(\dot{z}_{j}\right)+A_{0}\left(\dot{F}_{j}\right), \\
& \left(\dot{z}_{j}^{N}\right)_{t}=\sigma\left(\alpha_{j}\right) H_{h} D_{h} \dot{F}_{j}+A_{0}\left(\dot{z}_{j}\right)+A_{0}\left(\dot{F}_{j}\right) .
\end{aligned}
$$

By differentiating $\dot{F}_{j}$ in time and using the Bernoulli equation, we arrive at (see [3] for details)

$$
\left(\dot{F}_{j}\right)_{t}=-c\left(\alpha_{j}\right) \dot{z}_{j}^{N}+\frac{1}{2}\left(\dot{u}_{j}^{2}+\dot{v}_{j}^{2}\right)
$$

where $c\left(\alpha_{j}\right)=\left(u_{t}, v_{t}+g\right) \cdot \mathbf{n}$ is the net acceleration in the normal direction. When air is above water, the water wave problem is well-posed, and the sign condition, $c(\alpha)>0$, is satisfied (see [14]). In fact, we may assume that $c(\alpha) \geq c_{0}>0$.

As in [3], we will make a change of variables to eliminate the contribution from the tangential direction

$$
\dot{\delta}_{j}=\dot{z}_{j}^{T}+H_{h} \dot{z}_{j}^{N}, \quad \dot{z}_{j}^{N}=\dot{z}_{j}^{N}
$$


By differentiating $\dot{\delta}$ with respect to $t$ and using (39) and (40), we get after cancellation of the leading order terms that

$$
\begin{aligned}
\left(\dot{\delta}_{j}\right)_{t} & =A_{0}\left(\dot{z}_{j}\right)+A_{0}\left(\dot{F}_{j}\right), \\
\left(\dot{z}_{j}^{N}\right)_{t} & =\sigma\left(\alpha_{j}\right) H_{h} D_{h} \dot{F}_{j}+A_{0}\left(\dot{z}_{j}\right)+A_{0}\left(\dot{F}_{j}\right), \\
\left(\dot{F}_{j}\right)_{t} & =-c\left(\alpha_{j}\right) \dot{z}_{j}^{N}+\frac{1}{2}\left(\dot{u}_{j}^{2}+\dot{v}_{j}^{2}\right) .
\end{aligned}
$$

Thus, we have reduced the error estimates for the full nonlinear, nonlocal water wave equations into a simple linear and almost local system for the variation quantities. Now it is easy to perform an energy estimate based on the balance between $\dot{z}^{N}$ and $\dot{F}$, in the same way as in [3]. To illustrate the main idea, we will present the linear stability analysis below.

First, we observe that $H_{h} D_{h}$ is a symmetric positive operator. In fact a direct calculation shows that $H_{h} D_{h}$ has the discrete Fourier symbol $|k|(1-|k h| / \pi) \rho(k h)$, where $\rho(k h)$ is related to the Fourier symbol of $D_{h}$, i.e., ${\widehat{\left(D_{h}\right)}}_{k}=i k \rho(k h)$. By assumption, $\rho(k h) \geq 0$. Introduce a discrete $H^{1 / 2}$ norm

$$
\|f\|_{H_{h}^{1 / 2}}=\left(\left(H_{h} D_{h}+I\right) f, f\right)^{1 / 2}
$$

where $(f, g)=\sum_{j=1}^{N} f_{j} g_{j} h$ is the discrete inner product. To perform energy estimates, we multiply (42) by $\dot{\delta}_{j}$, (43) by $\frac{c\left(\alpha_{j}\right)}{\sigma\left(\alpha_{j}\right)} \dot{z}_{j}^{N}$, and (44) by $\left(H_{h} D_{h}+I\right) \dot{F}_{j}$, sum in $j$, then add up the resulting equations. Define

$$
y_{0}(t)^{2}=\left\|(c / \sigma)^{\frac{1}{2}} \dot{z}^{N}\right\|_{l^{2}}^{2}+\|\dot{\delta}\|_{l^{2}}^{2}+\|\dot{F}\|_{H_{h}^{1 / 2}}^{2},
$$

where $\|f\|_{l^{2}}^{2}=\sum_{j=1}^{N} f_{j}^{2} h$ is the discrete $L^{2}$ norm. It follows that

$$
\begin{aligned}
\frac{1}{2} \frac{d}{d t} y_{0}^{2}(t)= & \left(H_{h} D_{h} \dot{F}, c \dot{z}^{N}\right)-\left(c \dot{z}^{N},\left(H_{h} D_{h}+I\right) \dot{F}\right) \\
& +\left(f^{(1)}, \dot{z}^{N}\right)+\left(f^{(2)}, \dot{\delta}\right),
\end{aligned}
$$

where

$$
\left\|f^{(j)}\right\|_{l^{2}} \leq C\left(\left\|\dot{z}^{N}\right\|_{l^{2}}+\|\dot{\delta}\|_{l^{2}}+\|\dot{F}\|_{l_{2}}\right), \quad \text { for } j=1,2 .
$$

Here $C$ is a generic constant independent of $h$. This capital $C$ should not be confused with the little $c(\alpha)=\left(u_{t}, v_{t}+g\right) \cdot \mathbf{n}$. Note that the leading order terms in (45) cancel each other. The remaining terms are of lower order and can be bounded by $y_{0}^{2}$. Hence, we obtain

$$
\frac{1}{2} \frac{d}{d t} y_{0}^{2}(t) \leq C y_{0}^{2} .
$$

This proves linear stability. Nonlinear stability and convergence of the method can be obtained by using the asymptotic error expansion and Strang's argument [12] (also see [3]). Roughly speaking, Strang's observation is that if a numerical method is consistent with a well-posed (nonlinear) hyperbolic system and has an error expansion, then linear stability implies convergence as long as a smooth solution exists. The main idea is to construct a perturbation to the exact solution $z(\alpha, t)$,

$$
\tilde{z}(\alpha, t)=z(\alpha, t)+h z_{1}(\alpha, t)+h^{2} z_{2}(\alpha, t)+\cdots h^{m} z_{m}(\alpha, t),
$$

in such a way that $\tilde{z}(\alpha, t)$ satisfies the discrete equations up to high order, $O\left(h^{m+1}\right)$. The correction term $z_{j}$ can be determined recursively from $z, z_{1}, \ldots$, and $z_{j-1}$, and satisfies a linearized equation. The convergence analysis is then based on the error 
estimate for $\dot{z}_{j}=z_{j}(t)-\tilde{z}\left(\alpha_{j}, t\right)$ instead of $\dot{z}_{j}=z_{j}(t)-z\left(\alpha_{j}, t\right)$. By taking $m$ sufficiently large (say $m \geq 2$ in our case), the truncation error in $\dot{z}_{j}$ can be made arbitrarily small $\left(O\left(h^{m+1}\right)\right)$. Then nonlinear stability can be obtained by using the smallness of the truncation error. This implies convergence. We will omit the details here. This completes our stability analysis for the 2-D point vortex method.

In the above analysis, it is clear that the role of $B$ is to eliminate the destabilizing terms introduced by violating $H_{h}^{2} D_{h}=-D_{h}$. The specific form of this correction term can be explicitly determined after we project the errors into the tangent and normal coordinate and making the change of variable from $\dot{z}^{T}$ to $\dot{\delta}$.

\section{NumericAl RESUlts}

In this section, we present a series of numerical calculations to demonstrate the effect of various stabilizing terms in the stability analysis. We consider two examples. The first one is a standing wave. The second one is a breaking wave. The second example is a more severe test since it generates high frequency components dynamically. We found that for the standing wave, the point vortex method, without adding any stabilizing term, is numerically unstable. For this mild test problem, the first stabilizing correction, $A$, seems to be sufficient to stabilize the calculation for long time. However, for the breaking wave example, we found that we need both correction terms $A$ and $B$ in order to stabilize the calculation close to the breaking of the wave. This gives a strong numerical validation to our stability analysis.

In the standing wave calculations we use four different discretizations. In all cases, the velocity integral is discretized using the point vortex method. The space derivative $D_{\alpha}$ is approximated by the pseudo-spectral derivative with Fourier smoothing. In our computations, we choose a specific cut-off function

$$
\rho(x)=\left\{\begin{array}{cl}
1, & x \in[0, \pi / 2], \\
P\left(\frac{\pi-x}{\pi-x / 2}\right), & x \in(\pi / 2, \pi],
\end{array}\right.
$$

where $P(\xi)=35 \xi^{4}-84 \xi^{5}+70 \xi^{6}-20 \xi^{7}$. With this choice of $P(\xi)$, we have $\rho(\pi)=0$ and $\rho \in C^{3}[0, \pi]$. Since we use the same discrete derivative operator in all our four schemes, the only difference in these schemes is in the way we stabilize the spatial discretization of the velocity integral. In the first scheme, we use the new stabilizing technique which includes both correction terms $A$ and $B$. This scheme has been proved to be stable in Section 3. The second scheme uses only one stabilizing term $A$, but does not include $B$. The third scheme uses the Fourier filtering to enforce the compatibility condition $\Lambda_{h}\left(s^{p}\right)=H_{h} D_{h}(s)$, where $s$ is the periodic perturbation of $z$, i.e., $z(\alpha)=\alpha+s(\alpha), s^{p}$ is a Fourier filtering defined as $\left(\widehat{s}^{p}\right)_{k}=p(k h) \hat{s}_{k}$ for some cut-off function $p$ (please do not confuse $p$ with $\rho$ in the discrete derivative operator). The cut-off function $p$ is determined by satisfying the modified compatibility condition $\Lambda_{h}\left(s^{p}\right)=H_{h} D_{h}(s)$. For the point vortex method approximation, we can compute explicitly the Fourier symbols of $H_{h}$ and $\Lambda_{h}$. For 1-periodic functions, we have ${\widehat{\left(H_{h}\right)}}_{k}=-i \operatorname{sgn}(k)(1-2|k h|)$, and $\widehat{\left(\Lambda_{h}\right)_{k}}=2 \pi|k|(1-|k h|)$. Here $\operatorname{sgn}(x)$ is the sign function. The corresponding Fourier filtering is defined as $p(k h)=\rho(k h)(1-2|k h|) /(1-|k h|)$. Thus, our third scheme using the Fourier filtering is given as follows (after taking into account the 
1-periodicity)

$$
\begin{aligned}
\frac{d \bar{z}_{j}}{d t} & =\frac{1}{2 i} \sum_{k=1, k \neq j}^{N} \gamma_{k} \cot \left(\pi\left(z_{j}^{p}-z_{k}^{p}\right)\right) h+\frac{\gamma_{j}}{2 D_{h} z_{j}} \equiv u_{j}-i v_{j}, \\
\frac{d \phi_{j}}{d t} & =\frac{1}{2}\left(u_{j}^{2}+v_{j}^{2}\right)-g y_{j}, \\
D_{h} \phi_{j} & =\frac{1}{2} \gamma_{j}+\operatorname{Re}\left[\frac{D_{h} z_{j}}{2 i} \sum_{k=1, k \neq j}^{N} \gamma_{k} \cot \left(\pi\left(z_{j}^{p}-z_{k}^{p}\right)\right) h\right] .
\end{aligned}
$$

Finally, our fourth scheme is the point vortex method without using any stabilizing technique,

$$
\begin{aligned}
\frac{d \bar{z}_{j}}{d t} & =\frac{1}{2 i} \sum_{k=1, k \neq j}^{N} \gamma_{k} \cot \left(\pi\left(z_{j}-z_{k}\right)\right) h+\frac{\gamma_{j}}{2 D_{h} z_{j}} \equiv u_{j}-i v_{j}, \\
\frac{d \phi_{j}}{d t} & =\frac{1}{2}\left(u_{j}^{2}+v_{j}^{2}\right)-g y_{j}, \\
D_{h} \phi_{j} & =\frac{1}{2} \gamma_{j}+\operatorname{Re}\left[\frac{D_{h} z_{j}}{2 i} \sum_{k=1, k \neq j}^{N} \gamma_{k} \cot \left(\pi\left(z_{j}-z_{k}\right)\right) h\right] .
\end{aligned}
$$

In our calculations we choose a small sinusoidal perturbation to the equilibrium solution of period 1 . The initial condition is given by

$$
\begin{aligned}
& x(\alpha, 0)=\alpha+0.01 \sin (2 \pi \alpha), \quad y(\alpha, 0)=-0.01 \sin (2 \pi \alpha), \\
& \gamma(\alpha, 0)=0.01 \sin (2 \pi \alpha) .
\end{aligned}
$$

The gravity coefficient $g$ is set to be 10 . This choice of $g$ and the wavelength of the initial data, which is set to be 1 , determine the time and length scales. With this particular initial data, the familiar linear theory of water waves (see, e.g., [13]) predicts a standing wave, periodic in time, with period 0.801 .

Throughout our calculations we use a fourth-order explicit Adams-Bashforth method as our time integration scheme. A fourth-order explicit Runge-Kutta method is used to initialize the first three time steps in the Adams-Bashforth method. A simple iterative scheme is used to solve for $\gamma$. For example, for our first scheme, the $(m+1)$ st iterative solution for $\gamma$ is given by

$$
\begin{aligned}
& \gamma_{j}^{m+1}=2 D_{h} \phi_{j} \\
& -2 \operatorname{Re}\left(D_{h} z_{j}\left(\frac{1}{2 i} \sum_{k=1, k \neq j}^{N} \gamma_{k}^{m} \cot \left(\pi\left(z_{j}-z_{k}\right)\right) h+\frac{\gamma_{j}^{m}}{2 i\left(D_{h} z_{j}\right)^{2}}\left(\Lambda_{h}-H_{h} D_{h}\right) z_{j}\right)\right) .
\end{aligned}
$$

We use a fourth-order extrapolation in time to obtain a more accurate initial guess for $\gamma_{j}^{0}$ as suggested in [1. We stop the iteration when the difference between the two consecutive iterative solutions is smaller that $10^{-10}$.

In Figures 1a and 1b, we plot the numerical interface positions obtained from the first scheme from $t=0$ to $t=4.8$ and from $t=5.2$ to $t=10$, respectively. In these calculations, we use $N=128$ and $d t=0.0025$. As predicted from the linear theory, we obtain a standing wave with a period of about 0.8 time unit. So there are, in total, about 12.5 complete oscillations by time $t=10$. Clearly, the numerical solution is stable and smooth. It also suggests that there is a global smooth solution. 

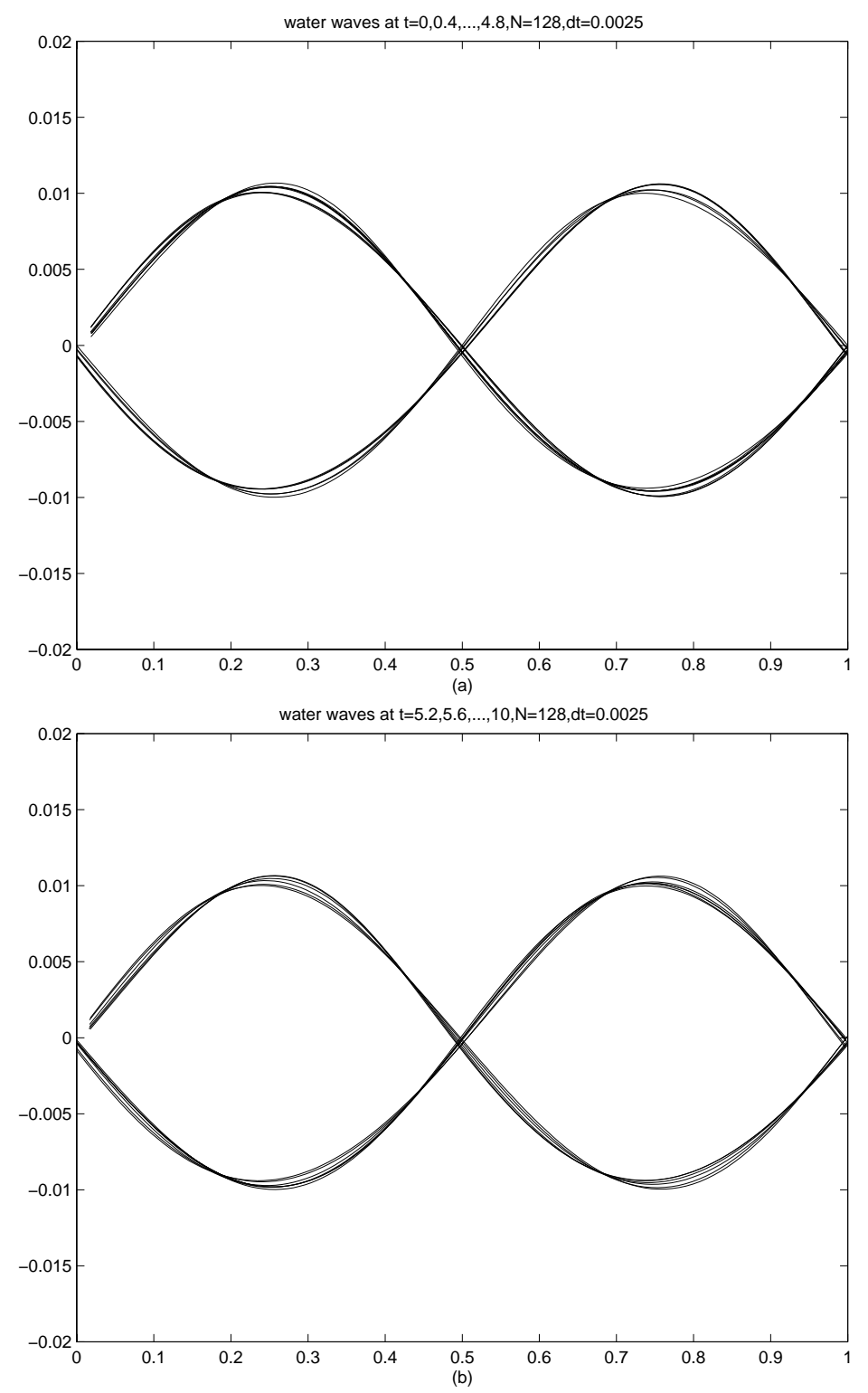

Figure 1. (a) Interface positions of the standing wave from $t=0$ to $t=4.8$ with time increment equal to 0.4 . Numerical solutions are obtained using the first scheme with $N=128$ and $\Delta t=0.0025$. (b) Interface positions of the standing wave from $t=5.2$ to $t=10$ with time increment equal to 0.4 . The same computation as in Figure 1(a).

In Figure 2, we plot $\log \left|\hat{s}_{k}\right|$, where the $\log$ is the natural logarithm. We can see that the round-off errors remain small at all times, indicating that no high-mode instability occurs in the calculation. We also test the time step stability in our computations. In Figure 11, we plot the maximum sizes of the time steps allowed 


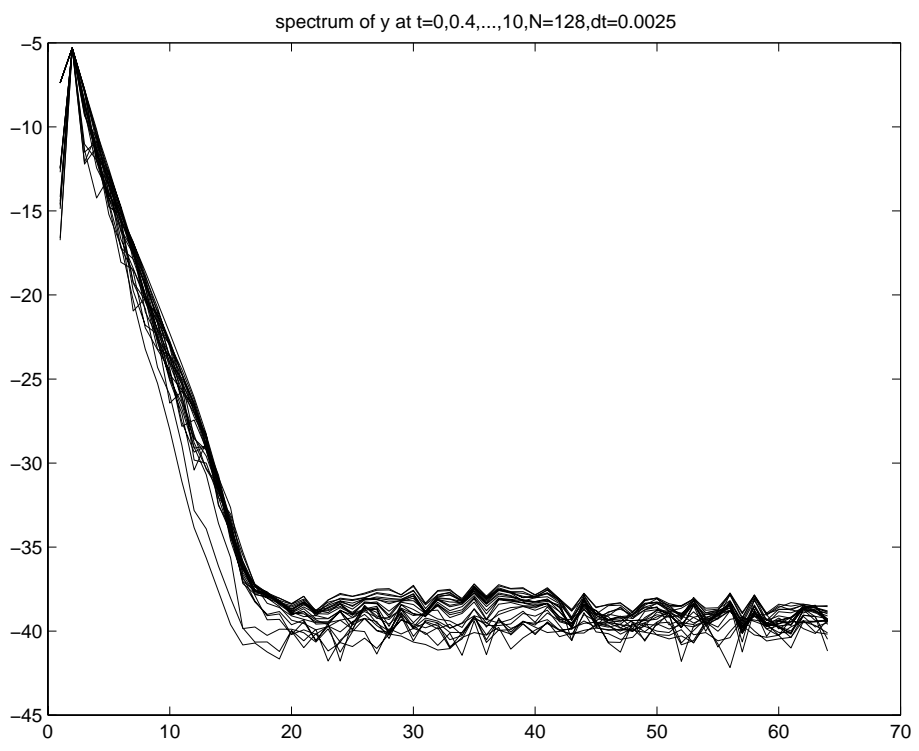

FIGURE 2. $\log \left|\hat{y}_{k}\right|$ versus the wavenumbers $k$ from $t=0$ to $t=10$ time increment equal to 0.4. Numerical solutions are obtained using the first scheme with $N=128$ and $\Delta t=0.0025$.

for obtaining time step stability for different mesh sizes. The time step and the mesh size clearly satisfy a linear CFL condition, i.e. $\Delta t \leq C h$.

The calculations presented in Figures 3 and 4 are for the second scheme and the third scheme, respectively. The numerical solutions corresponding to these calculations appear to be stable, at least up to the time we have computed. The two schemes give almost indistinguishable solutions. This suggests that the numerical instability caused by violating the compatibility condition $H_{h}^{2} D_{h}=-D_{h}$ is much milder than that caused by violating the compatibility condition $\Lambda_{h}=H_{h} D_{h}$. This is expected. In fact, the equilibrium stability analysis shows that the method is stable as long as the compatibility condition $\Lambda_{h}=H_{h} D_{h}$ is satisfied.

The calculations presented in Figures 5 and 6 are for the point vortex method without any stability technique. In Figure 5, we plot the vortex sheet strength. The numerical solution has developed oscillations by this time. Moreover, the numerical oscillation grows rapidly in time. Increasing the numerical resolution does not help to reduce the numerical instability. In fact, the oscillation will develop earlier with increasing resolutions, indicating numerical instability. In Figure 6 we examine the growth of the Fourier coefficients associated with the vortex strength. The log plot of the spectrum at different times is illustrated in Figure 6. For $t$ small, we see that the spectrum decays exponentially. But as time increases, the round-off errors are amplified by the numerical instability. As we can see, the round-off errors at the high modes are amplified the fastest. By the time the high frequency modes have grown to order $O(1)$, we observe order one oscillations in the physical space. We remark that using the $\gamma$ formulation (10) gives a more stable discretization than the corresponding formulation for the dipole strength $\mu$. The growing eigenvalues for the $\gamma$ formulation are at most of order $O(k)$, whereas the growing eigenvalues for the dipole formulation can be as large as $O\left(k^{2}\right)$. 

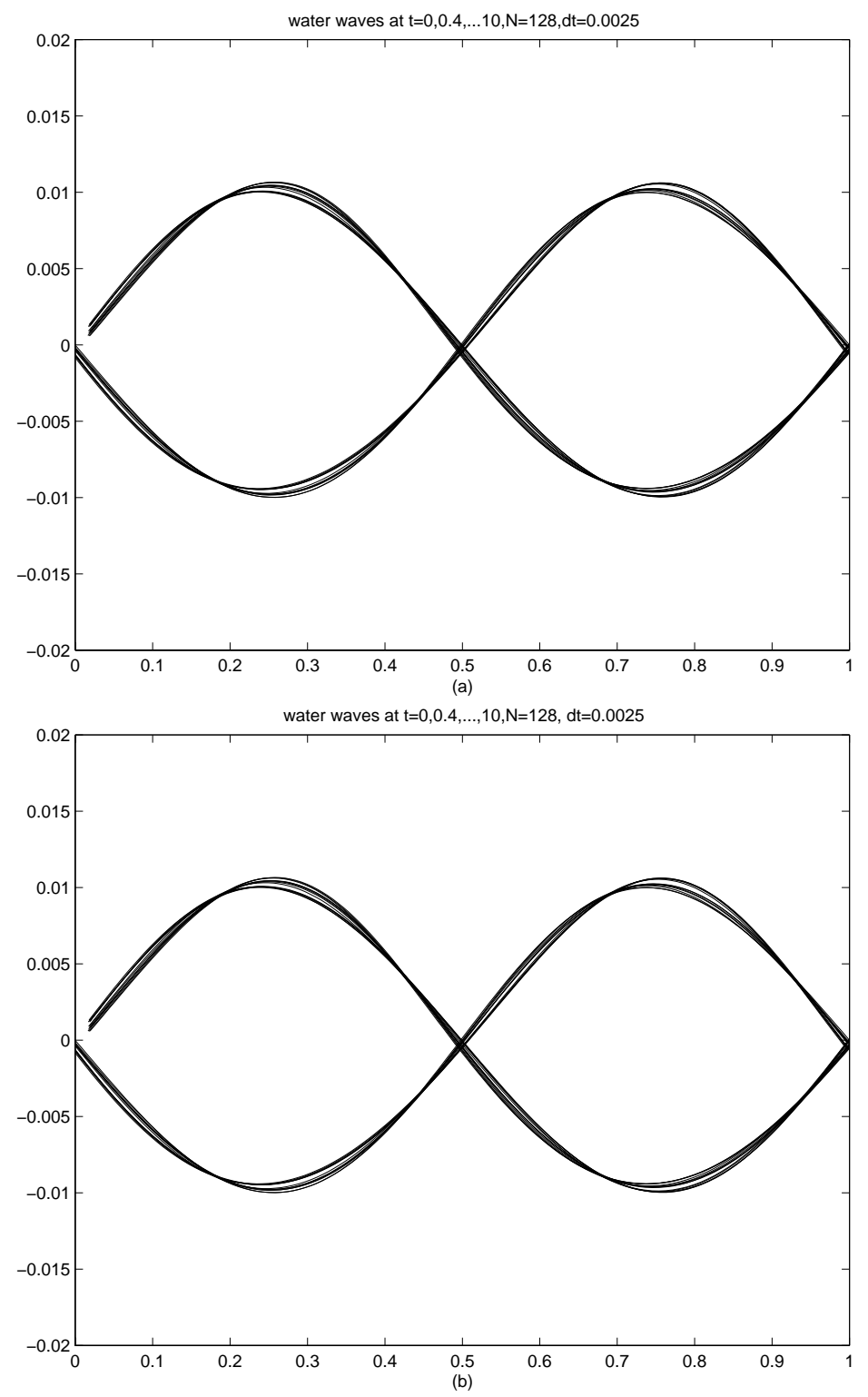

FiguRE 3. (a) Interface positions of the standing wave from $t=0$ to $t=10$ with time increment equal to 0.4 . Numerical solutions are obtained using the second scheme with $N=128$ and $\Delta t=0.0025$. (b) Interface positions of the standing wave from $t=0$ to $t=10$ with time increment equal to 0.4 . Numerical solutions are obtained using the third scheme with $N=128$ and $\Delta t=0.0025$.

Next, we present calculations of the breaking wave using the point vortex method. In order to produce breaking in the water wave we use the initial condition

$$
\begin{aligned}
& x(\alpha, 0)=\alpha, \quad y(\alpha, 0)=0.1 \cos (2 \pi \alpha), \\
& \gamma(\alpha, 0)=-1.0+0.1 \sin (2 \pi \alpha) .
\end{aligned}
$$



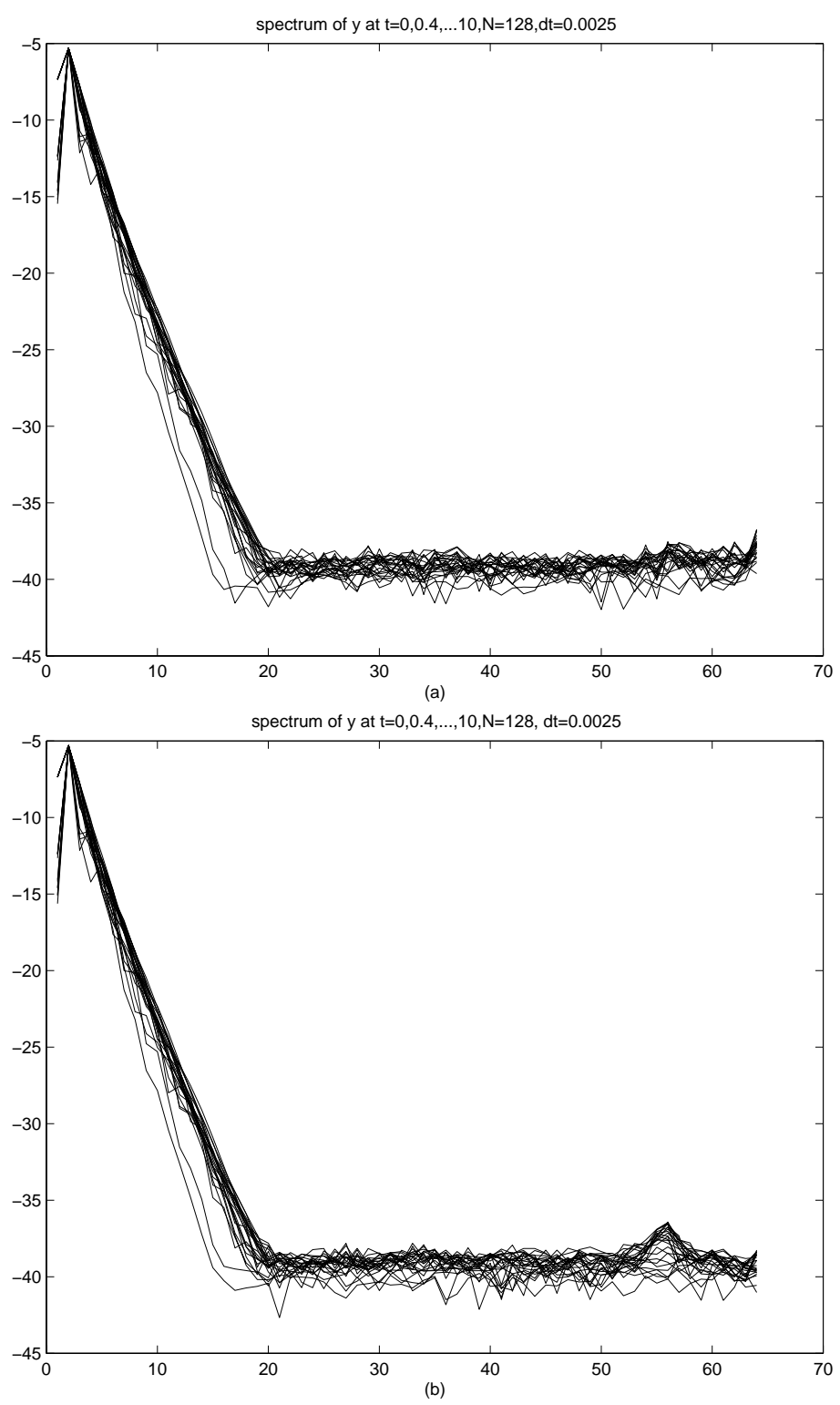

Figure 4. (a) $\log \left|\hat{y}_{k}\right|$ versus the wavenumbers $k$ from $t=0$ to $t=$ 10 time increment equal to 0.4. Numerical solutions are obtained using the second scheme with $N=128$ and $\Delta t=0.0025$. (b) $\log \left|\hat{y}_{k}\right|$ versus the wavenumbers $k$ from $t=0$ to $t=10$ time increment equal to 0.4. Numerical solutions are obtained using the third scheme with $N=128$ and $\Delta t=0.0025$.

Note that the vortex sheet strength $\gamma$ does not have zero mean in this case. With this choice of initial data, there is a shear velocity at infinity equal to $\pm 1 / 2$.

In Figure 7, we present two numerical calculations with $N=256$ and $N=512$ using the first scheme in which both correction terms are included. With $N=512$, 

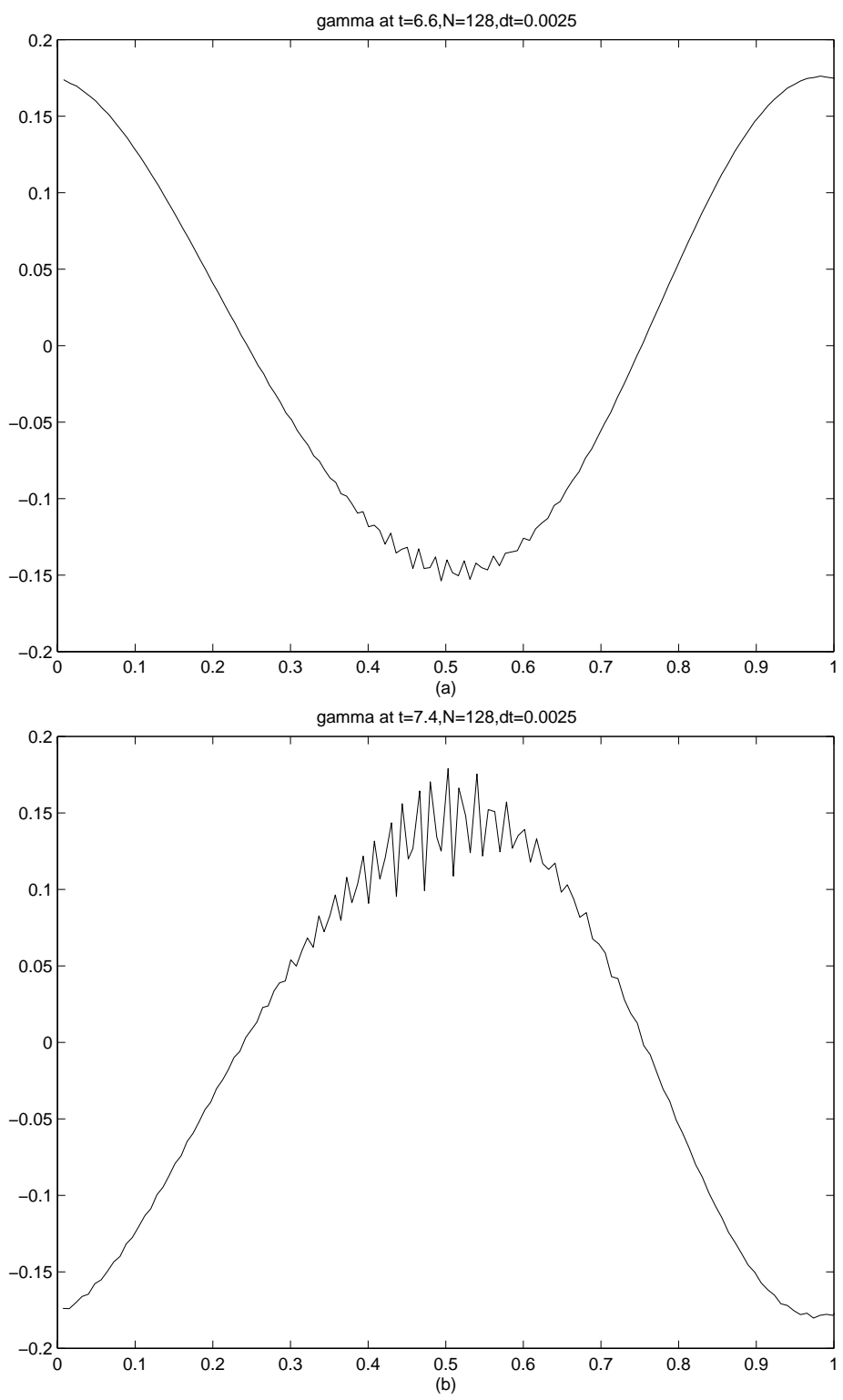

Figure 5. (a) Vortex sheet strength $\gamma(\alpha, t)$ as a function $\alpha$ at $t=6.6$. The numerical solution is computed using the fourth scheme with $N=128$ and $\Delta t=0.0025$. (b) Vortex sheet strength $\gamma(\alpha, t)$ as a function $\alpha$ at $t=7.4$. The same computation as in Figure 5(a).

we can compute longer in time due to the increase of numerical resolution. In Figure 8, curvatures at different times are also presented. In Figure 9, we plot the Fourier spectrum of the interface positions (the $y$ component). It is clear that the numerical round-off errors are kept small even in the fully nonlinear regime of the solution. Again, we test the time step stability in our computations. In Figure 


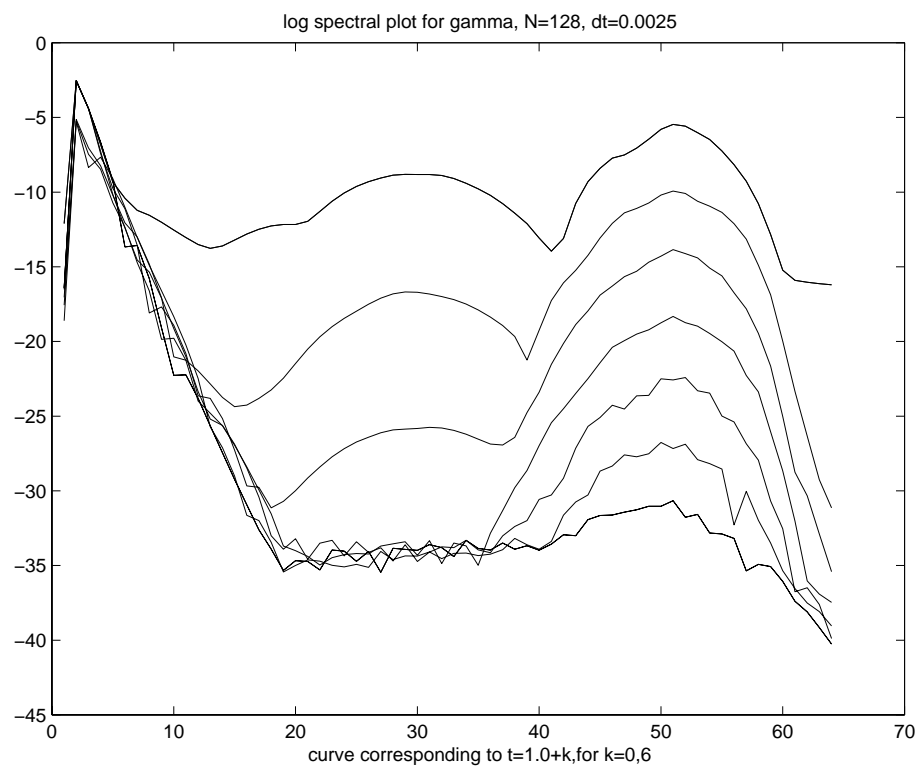

FiguRE $6 . \log \left|\hat{\gamma}_{k}\right|$ versus the wavenumbers $k$ from $t=1$ to $t=4.6$ time increment equal to 0.6. Numerical solutions are computed using the fourth scheme with $N=128$ and $\Delta t=0.0025$.

12, we plot the maximum sizes of the time steps allowed for obtaining time step stability for different mesh sizes. The time step and the mesh size clearly satisfy a linear CFL condition, i.e., $\Delta t \leq C h$.

We repeat the same calculation using the second and the third schemes. Neither scheme enforces the compatibility condition, $\left(H_{h}^{2}+I\right) D_{h}=0$. Stability analysis near equilibrium does not reveal any unstable eigenvalues, but our stability analysis far from equilibrium reveals the potential numerical instability. The inclusion of the second stabilizing term $B$ is exactly to eliminate this potential numerical instability induced by violating the compatibility condition $\left(H_{h}^{2}+I\right) D_{h}=0$. Our numerical computations indicate that both schemes give rise to numerical instabilities in the breaking wave calculations. In Figure 10, we plot the Fourier spectrum of the interface position (the $y$ component) for both schemes. It is evident that round-off errors grow rapidly in time, leading to large oscillations in the physical space at later times. It is also interesting to note that the numerical instabilities seem to occur at almost the same time for the both calculations. 

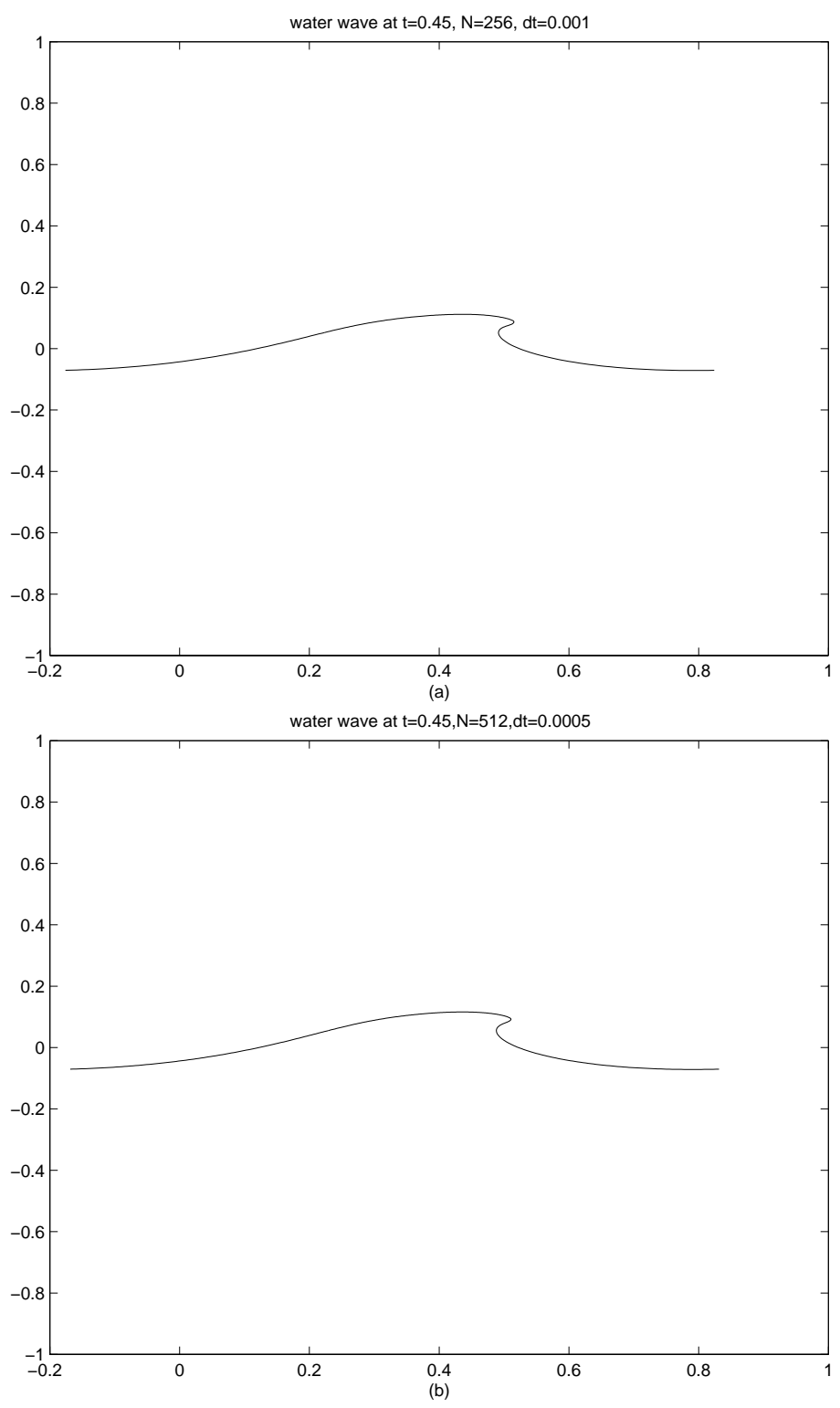

FiguRE 7. (a) Interface position of the breaking wave at $t=0.45$ computed from the first scheme with $N=256$ and $\Delta t=0.001$. (b) The same calculation as in Figure 7 (a) with $N=512$ and $\Delta t=0.0005$. 

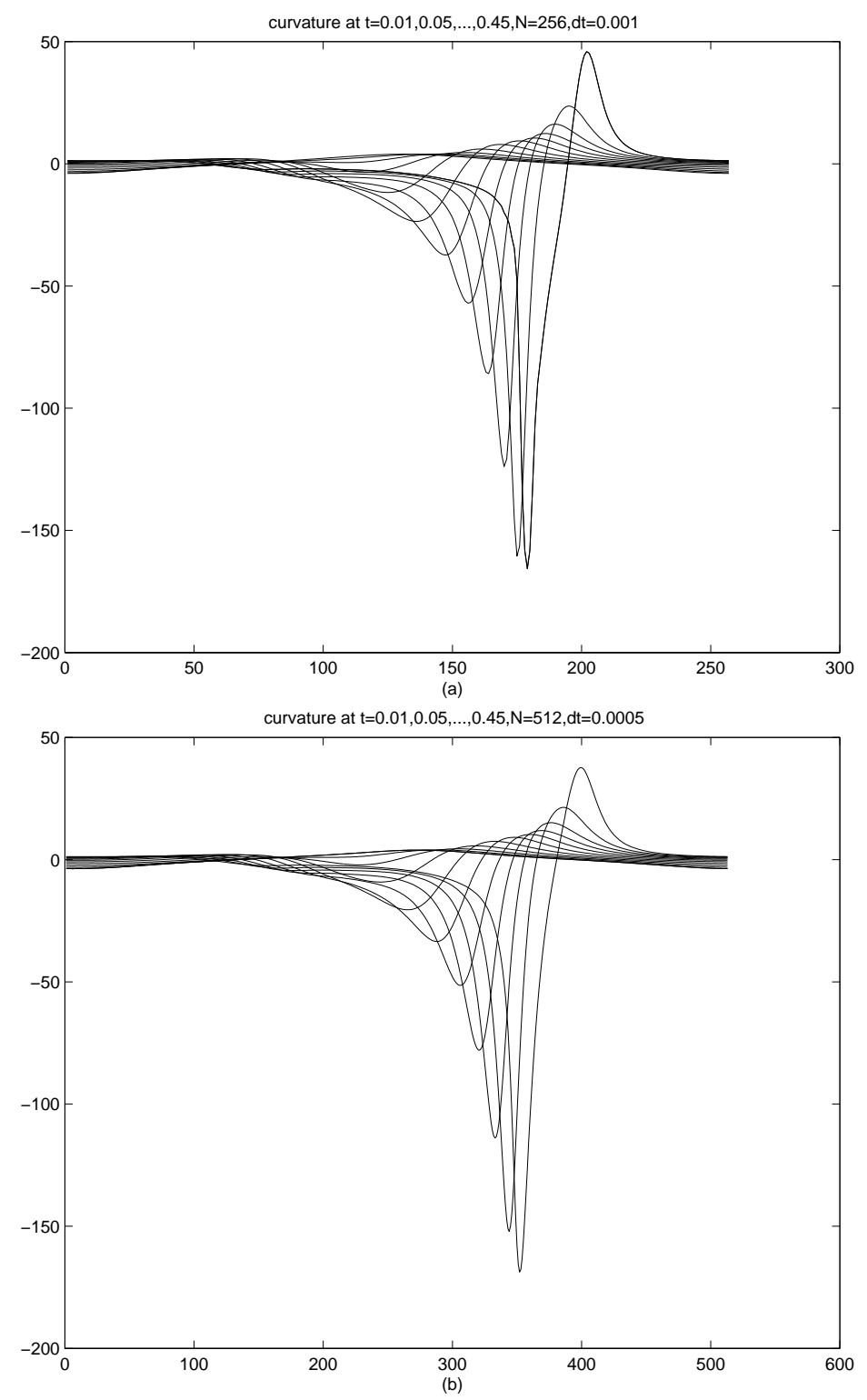

Figure 8. (a) Curvatures of the breaking wave from $t=0.01$ to 0.45 with time increment equal to 0.04. Solutions are computed from the first scheme with $N=256$ and $\Delta t=0.001$. (b) The same calculation as in Figure 8(a) with $N=512$ and $\Delta t=0.0005$. 

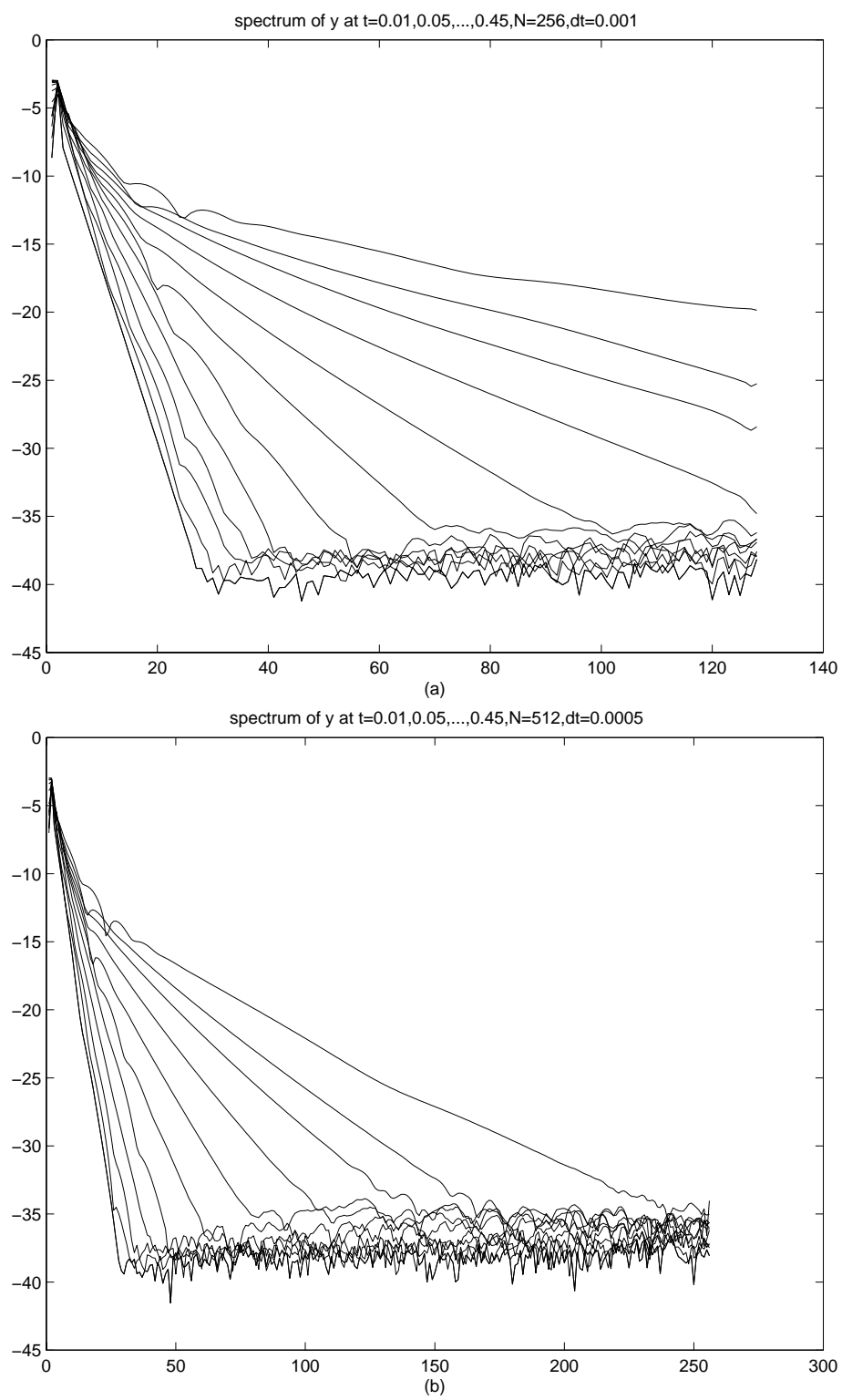

Figure 9. (a) $\log \left|\hat{y}_{k}\right|$ versus the wavenumbers $k$ from $t=0.01$ to $t=0.45$ time increment equal to 0.04 . Numerical solutions are obtained using the first scheme with $N=256$ and $\Delta t=0.001$. (b) The same calculation as in Figure $9($ a) with $N=512$ and $\Delta t=$ 0.0005 . 

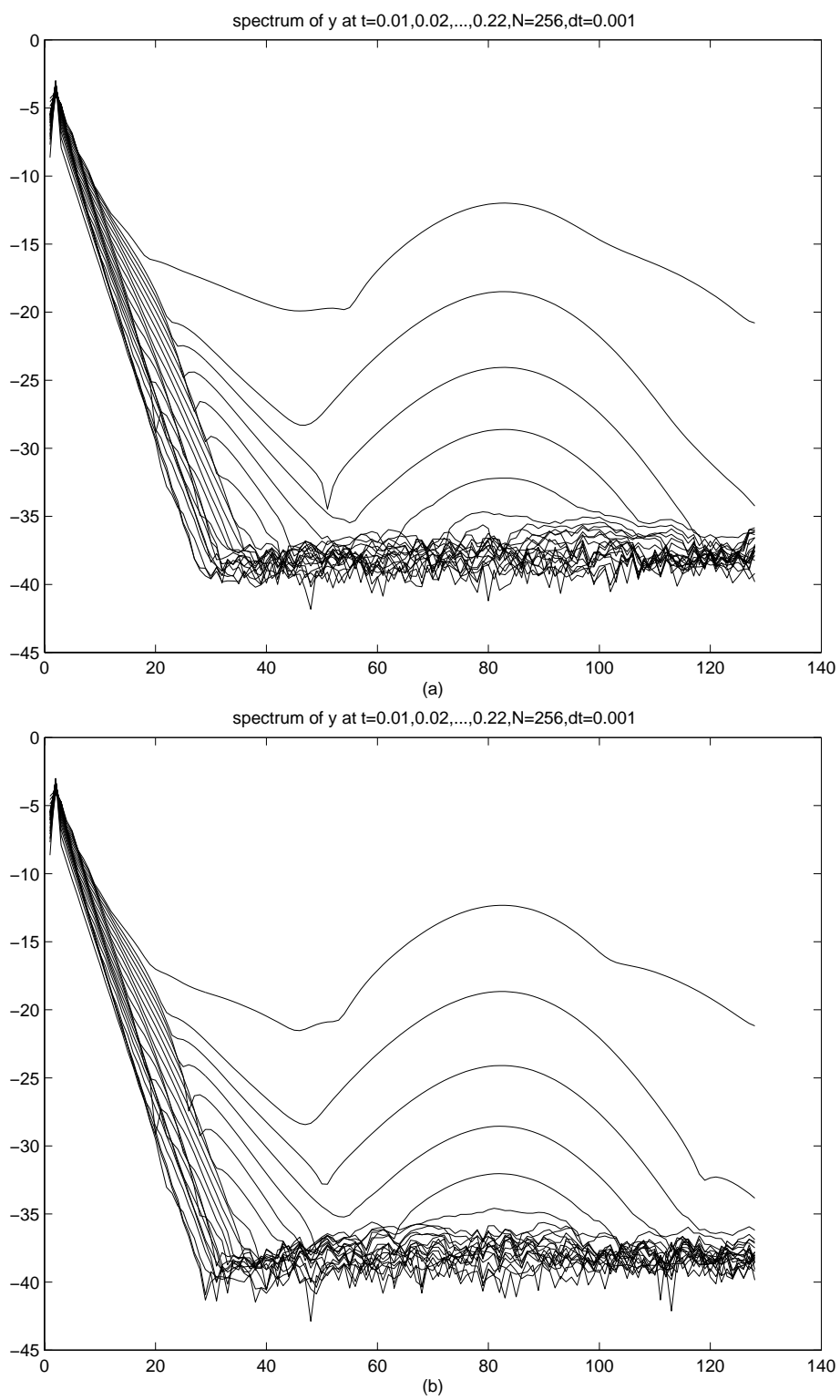

Figure 10. (a) $\log \left|\hat{y}_{k}\right|$ versus the wavenumbers $k$ from $t=0.01$ to $t=0.22$ time increment equal to 0.01 . Numerical solutions are obtained using the second scheme with $N=256$ and $\Delta t=0.001$. (b) $\log \left|\hat{y}_{k}\right|$ versus the wavenumbers $k$ from $t=0.01$ to $t=0.22$ time increment equal to 0.01. Numerical solutions are obtained using the third scheme with $N=256$ and $\Delta t=0.001$. 


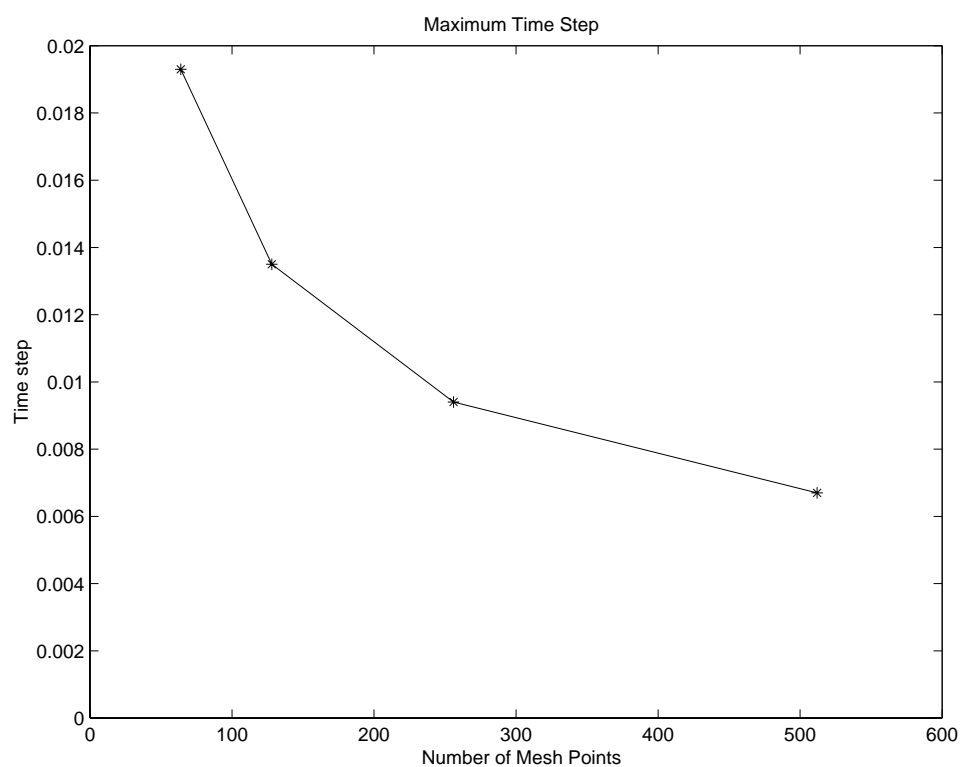

Figure 11. The maximum sizes of the time steps versus the number of mesh points. Computations are performed for the first scheme for the standing water wave.

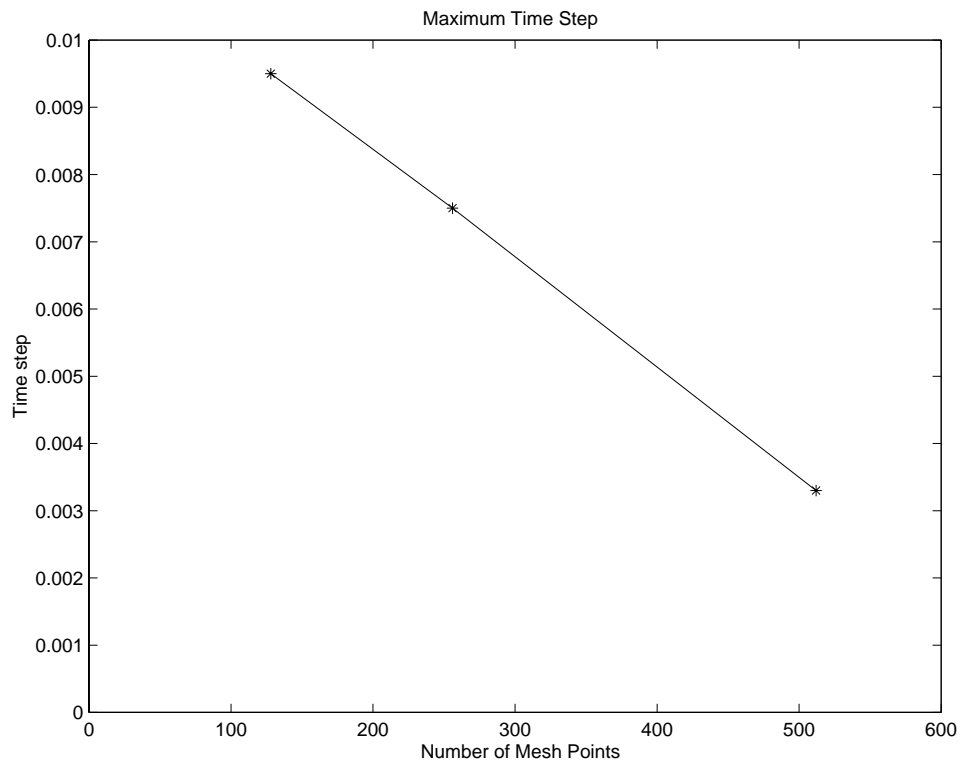

Figure 12. The maximum sizes of the time steps versus the number of mesh points. Computations are performed for the first scheme for the breaking water wave. 


\section{ACKNOWLEDGMENTS}

We would like to thank Dr. Hector Ceniceros for several valuable suggestions during the preparation of this paper. We also appreciate constructive comments from the referee.

\section{REFERENCES}

[1] G. Baker, D. Meiron, and S. Orszag, Generalized vortex methods for free-surface flow problems, J. Fluid Mech. 123, 477-501 (1982). MR 84a:76002

[2] G. Baker, and A. Nachbin, Stable methods for vortex sheet motion in the presence of surface tension, SIAM J. Sci Comput., 19, 1737-1766 (1998). MR 99c:76073

[3] J.T. Beale, T.Y. Hou and J.S. Lowengrub, Convergence of a boundary integral method for water waves, SIAM J. Numer. Anal., 33, 1797-1843 (1996). MR 98b:76009

[4] R. E. Caflisch and J. S. Lowengrub, Convergence of the Vortex Method for Vortex Sheets, SIAM J Numer. Anal., 26, 1060-1080 (1989). MR 91g:76073

[5] J. W. Dold, An efficient surface-integral algorithm applied to unsteady gravity waves, J. Comp. Phys., 103, 90-115 (1992). MR 93g:76091

[6] J. Goodman, T. Y. Hou and J. Lowengrub, Convergence of the point vortex method for the 2-D Euler equations, Comm. Pure and Appl. Math., 43, 415-430 (1990). MR 91d:65152

[7] T. Y. Hou, Numerical Solutions to Free Boundary Problems, Acta Numerica, 335-415 (1995). MR 96i:65113

[8] T. Y. Hou, Z.-H. Teng, and P. Zhang, Well-Posedness of Linearized Motion for 3-D Water Waves Far From Equilibrium, Comm. in PDE's, 21, 1551-1586 (1996). MR 98c:76013

[9] A. I. Markushevich, Theory of Functions of a Complex Variable, translated to English by Richard Silverman, second edition, Chelsea Publishing Company, New York, 1977. MR 56:3258

[10] A.J. Roberts, A stable and accurate numerical method to calculate the motion of a sharp interface between fluids, I.M.A. J. Appl. Math 31, 13-35 (1983).

[11] L. Rosenhead, The point vortex approximation of a vortex sheet, Proc. Roy. Soc. London Ser. A, 134, 170-192 (1932).

[12] G. Strang, Accurate partial differential methods II: Nonlinear problems, Numer. Math., 6, 37-64 (1964). MR 29:4215

[13] G. B. Whitham, Linear and Nonlinear Waves, John Wiley \& Sons. Publications, New York, 1974. MR 58:3905

[14] S. J. Wu, Well-posedness in Sobolev spaces of the full water-wave problem in 2-D, Invent. Math., 130, 39-72 (1997). MR 98m:35167

Applied Mathematics, California Institute of Technology, Pasadena, California 91125

E-mail address: hou@ama.caltech.edu

School of Mathematical Science, Peking University, Beijing 100871, China

E-mail address: pzhang@sxx0.math.pku.edu.cn 